\title{
ARTICLE \\ Quantum Algorithm of Imperfect KB Self-organization Pt I: Smart Control-Information-Thermodynamic Bounds
}

\section{S.V. Ulyanov*}

Department of Quantum Control and Quantum Software Engineering, JINR, LIT; State University Dubna, Russia

\begin{tabular}{|c|c|}
\hline ARTICLE INFO & ABSTRACT \\
\hline Article history & \multirow{9}{*}{$\begin{array}{l}\text { The quantum self-organization algorithm model of wise knowledge } \\
\text { base design for intelligent fuzzy controllers with required robust level } \\
\text { considered. Background of the model is a new model of quantum inference } \\
\text { based on quantum genetic algorithm. Quantum genetic algorithm applied } \\
\text { on line for the quantum correlation's type searching between unknown } \\
\text { solutions in quantum superposition of imperfect knowledge bases of } \\
\text { intelligent controllers designed on soft computing. Disturbance conditions } \\
\text { of analytical information-thermodynamic trade-off interrelations between } \\
\text { main control quality measures (as new design laws) discussed in Part I. } \\
\text { The smart control design with guaranteed achievement of these trade- } \\
\text { off interrelations is main goal for quantum self-organization algorithm } \\
\text { of imperfect KB. Sophisticated synergetic quantum information effect } \\
\text { in Part I (autonomous robot in unpredicted control situations) and II } \\
\text { (swarm robots with imperfect KB exchanging between "master - slaves") } \\
\text { introduced: a new robust smart controller on line designed from responses } \\
\text { on unpredicted control situations of any imperfect KB applying quantum } \\
\text { hidden information extracted from quantum correlation. Within the toolkit } \\
\text { of classical intelligent control, the achievement of the similar synergetic } \\
\text { information effect is impossible. Benchmarks of intelligent cognitive } \\
\text { robotic control applications considered. }\end{array}$} \\
\hline Received: 29 April 2021 & \\
\hline Accepted: 28 June 2021 & \\
\hline Published Online: 15 July 2021 & \\
\hline Keywords: & \\
\hline Quantum genetic algorithm & \\
\hline & \\
\hline Quantum inference & \\
\hline Intelligent cognitive robotics & \\
\hline
\end{tabular}

\section{Introduction}

According to the definition of modern control problems, the achievement of required robustness property for a complex ill-defined control object models is possible with applying the computational intelligence toolkit. The goal of this article is the description of the applied aspects of developed intelligent design technology of robust knowledge bases $(\mathrm{KB})^{[1-7]}$ using the information synergy effects of quantum knowledge self-organization ${ }^{[2]}$ in unpredicted and risk control conditions ${ }^{[5]}$.
Physical extractable maximal amount work and the information source correlation between the system and the memory. The maximal amount work extractable from the system is bound by the nonequilibrium free energy change ${ }^{[8,9]}$. Entropy and correlation reversely related. Work can be extracted from the correlation between subsystems and the mutual memory of correlations ${ }^{[10]}$. Dissipative information amount characterizes a waste of such correlation work. Thus, positive quantum dissipative information characterizes a potential work waste and the information related to the environments is considered as lost. The

*Corresponding Author:

S.V. Ulyanov,

Department of Quantum Control and Quantum Software Engineering, JINR, LIT; State University Dubna, Russia;

Email:ulyanovsv@mail.ru 
conditional mutual information can be regarded as a part of the entropy production. The conditional free energy is equal to the maximal extractable work from the system given the memory information amount. In other words, more work can be extracted from the correlation between the system and the memory if the correlation is accessible ${ }^{[11]}$. Considered statements are the physical source background for design of considered quantum algorithm based on work done applying extractable hidden quantum information amount from classical control states.

Information-thermodynamic background of self-organization quantum algorithm. Classical states of dynamic processes include hidden quantum information that considered as additional information source for the compensation of value work waste. It is possible produce additional control action with extractable work and hidden quantum amount information in classical states is the resource of this value work. Information is physical and applied for the implementation of self-organization processes in intelligent control system using quantum - classical correlation between classical states. Four statements from quantum information theory and quantum thermodynamics are applied in this developed approach: 1) minimum entropy production rate principle of the system "control object + intelligent controller" that quadrantes the achievement of control goal with minimum of work waste in control object and in intelligent controller; 2) minimum information entropy principle for design intelligent cognitive controller that required minimum of initial information for intelligent controller action; 3 ) the amount of the work wasted on the extraction of hidden quantum information is less than the amount of work done on the received extracted quantum hidden information; and 4) the solution problem search of maximum extractable value work identical to a search of the minimum wasted entropy done on this work extraction. (see in details Appendix $1^{[4-12]}$ ).

The article task (as the continuation of ${ }^{[1-7]}$ ) is the description of the IT-design processing a robust sophisticated $\mathrm{KB}$ of intelligent cognitive controller that produces control force that is satisfied to these requirements.

\section{Problem Statement: Main Tasks}

The development and applications computational intelligence toolkit is the background of the end-to-end quantum computing technology ${ }^{[2,5,13]}$. As a consequence, one of subproblems is the creation for practical quantum algorithm realization (in on line) the process correction of time-dependent parameters of conventional PID controllers for its adaptation and robustness achievement in on line.

Remark. Many control theoretic algorithms augment the basic delta rule with additional control terms that greatly improve accuracy, stability, and responsivity. The most popular variant of these control theoretic models is the popular proportional-integral-derivative (PID) controller. This model is simple, accurate, and robust, with response properties that have been well characterized over the last century. The PID controller takes the error from a reference signal as input, and it outputs a control signal consisting of a linear combination of control signals proportional to the error (P-Term), the integral of the error (I-Term), and the derivative of the error (D-Term). These three terms minimize deviations from the reference based on errors in the present, past, and expected future, respectively. Proportional control (cf. delta-rule control) directly minimizes deviation from the reference and is often the primary driver of the control process. Integral control provides low-frequency compensation for residual steadystate errors, allowing the controller to reduce noise and track gradual changes in the environment. Derivative control provides high-frequency compensation that increases stability, such as by dampening control adjustments when the controller is approaching the reference or increasing adjustments when the reference or environment suddenly changes. Intuitively, integral control provides low-frequency compensation by combining several time points, whereas derivative control provides high-frequency compensation by tracking the instantaneous change. To behave adaptively in environments that are noisy and nonstationary, humans and other animals must monitor feedback from their environment and adjust their predictions and actions accordingly. An understudied approach for modeling these adaptive processes comes from the engineering field of control theory, which provides general principles for regulating dynamical systems, often without requiring a generative model. The proportional-integral-derivative (PID) controller is one of the most popular models of industrial process control. The proportional term is analogous to the "delta rule" in psychology, adjusting estimates in proportion to each error in prediction. The integral and derivative terms augment this update to simultaneously improve accuracy and stability. Here, it tested whether the PID-controller with time-dependent coefficient gain schedule can describe how people sequentially adjust their predictions of control object in response to new information.

Problem statement. Principle of the minimum of the generalized entropy of the system ("control object + conventional controller") considered as the fitness function in quantum genetic algorithm (QGA) and considered as a computed robustness measure). QGA-model embedded in the structure of quantum search algorithm and search 
quantum types of correlation between solutions in quantum superposition (of classical control states that designed with soft computing technology). The information technology (IT) of KB remote design of the fuzzy controllers developed on the base of the "Soft / Quantum computing KB optimizer" software toolkit (SCOptKB \& QSCOptKB) ${ }^{\mathrm{TM}}$. Mathematical background of the toolkit is quantum soft computing ${ }^{[5,13]}$. In Parts I and II applied Benchmarks of the developed IT design in detail discussed. In particular cases, in the design stage framework of robust KBs applying the model of quantum fuzzy inference (QFI): two different models of robots - mobile manipulator and inverted swing pendulum ("cart - pole" system) are shown.

A comparison of the quality control in the fuzzy controllers and quantum fuzzy controller in various control modes presented. The ability to connect and work with a real control object, without using his mathematical model described. The implemented technology of knowledge sharing in a swarm of intelligent robots, with quantum controllers, allows to achieve the goal of control and to gain additional knowledge by creating a new information source based on the synergetic effect of combining knowledge. The results of the experiments demonstrate the possibility of the ensured achievement of the control goal of a group of robots applying end-to-end quantum computing technology design of KBs of smart controllers in control system loop. The developed software toolkit allows to design and setup complex ill-defined and weakly formalized technical systems on line.

In Part I we consider the sophisticated structure of quantum control algorithm as the background for the QFI model design in Part II.

\section{Imperfect KB Quantum Self-organization Process}

The role of specific quantum hidden information effects for smart control design described in ${ }^{[2]}$. The amount of hidden quantum information ${ }^{[2,3,4,6]}$ extracted from control classical states considered as the additional information-thermodynamic control force source.

\subsection{Quantum Supremacy Effects of Imperfect KB Self-organization}

In natural systems the sought robustness property is coded in the algorithm of reproduction of the self-organization process ${ }^{[1,2]}$. The man-made quantum self-organization control process of the robustness property achievement of the $\mathrm{KB}$ is demonstrated in Figure 1.

Distributed under creative commons license 4.0

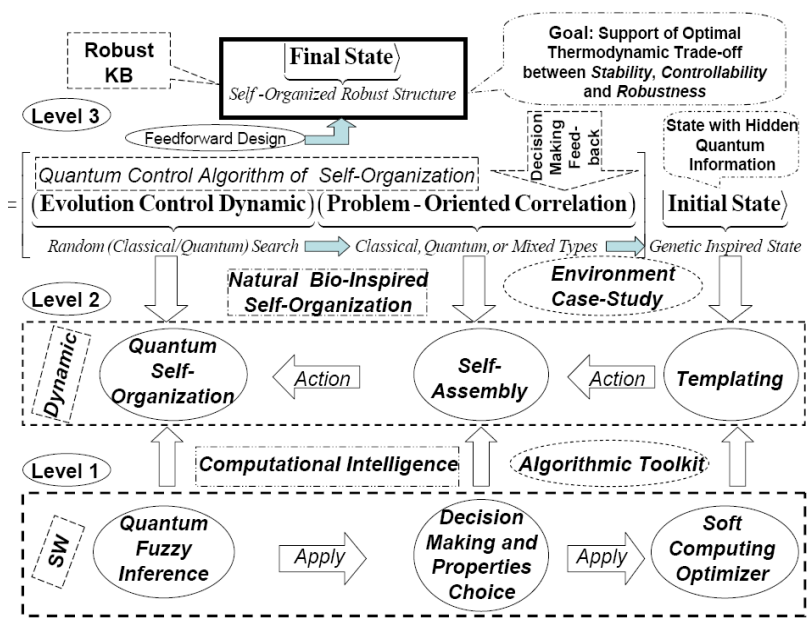

Figure 1. Quantum search hierarchical structure of self-organization robust KB's design system

Natural evolution processes are based on the following steps: (i) templating; (iii) self-assembling; and (iii) self-organization (level 2 in Figure1).

Remark. Analysis of self-organization models on Figure1 gives us the following results. Models of self-organization are included natural quantum effects and based on the following information-thermodynamic concepts: (i) macroand micro-level interactions with information exchange (in agent-based models - ABM - micro-level is the communication space where the inter-agent messages are exchange and is explained by increased entropy on a micro-level); (ii) communication and information transport on micro-level ("quantum mirage" in quantum corrals); (iii) different types of quantum spin correlation that design different structure in self-organization (quantum dot); (iv) coordination control (as example, swam-bot and snake-bot).

According quantum computing theory in general form every quantum algorithm (QA) includes the following unitary quantum operators: (i) superposition; (ii) entanglement (quantum oracle); (iii) interference. Measurement is the fourth classical operator. [It is irreversible operator and is used for measurement of computation results].

Quantum control algorithm of self-organization that developed in Figure 1 (level 1) is based on quantum fuzzy inference (QFI) models. QFI includes these concepts of self-organization and has realized by corresponding quantum operators.

QFI is one of possible realization of quantum control algorithm of self-organization that includes all of these features: (i) superposition; (ii) selection of quantum correlation types; (iii) information transport and quantum oracle; and (iv) interference. With superposition is realized templating operation, and based on macro- and micro-level interactions with information exchange of active 
agents. Selection of quantum correlation type organize self-assembling using power source of communication and information transport on micro-level. In this case the type of correlation defines the level of robustness in designed KB of FC. Quantum oracle calculates intelligent quantum state that includes the most important (value) information transport for coordination control. Interference is used for extraction the results of coordination control and design in on-line robust KB.

The developed QA of self-organization is applied to design of robust $\mathrm{KB}$ of $\mathrm{FC}$ in unpredicted control situations. Main operations of developed QA and concrete examples of QFI applications are described.

Fulfillment of requirements in the information - thermodynamic criteria (see Figure 1, level 3) guarantee in on line invariant control objective reaching of smart control with the required robustness level.

The corresponding design process shown in ${ }^{[2,4]}$ as following:

$$
\begin{aligned}
& \text { |Designed self - organized robust state } \\
& =\left[\begin{array}{l}
\left(\begin{array}{l}
\begin{array}{l}
\text { Evolution of self- } \\
\text { organization process }
\end{array}
\end{array}\right) \cdot\left(\begin{array}{l}
\text { Quantum } \\
\text { computing }
\end{array}\right) \cdot \underbrace{\underbrace{\text { Problem orientation of control object }}_{\text {Classical, quantum, mixed }}}_{\text {Quantum random search }}
\end{array}\right] \\
& \left\{\begin{array}{l}
\text { Initialstate }\rangle \\
\text { "Building" } \\
\text { block }
\end{array}\right\} \bullet\left(\begin{array}{l}
\text { Bio-inspired } \\
\text { states reproduced } \\
\text { by toolkit }
\end{array}\right) \text {, }
\end{aligned}
$$

Let us consider particular case (1).

\subsection{Quantum Self-organization of Imperfect KBs}

For design of production rules of $\mathrm{KB}$ in intelligent hybrid PID controller the structure of corresponding quantum algorithm has the following form from (1) [in Figure 1 (level 3)]:

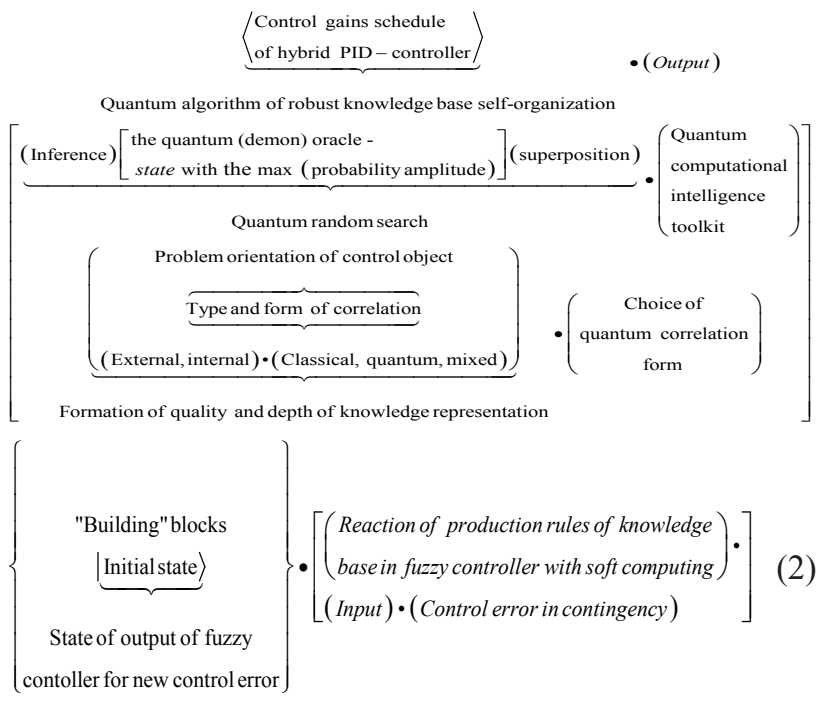

Figure 2 demonstrates the structure of robust intelligent control system based on quantum fuzzy inference (QFI).

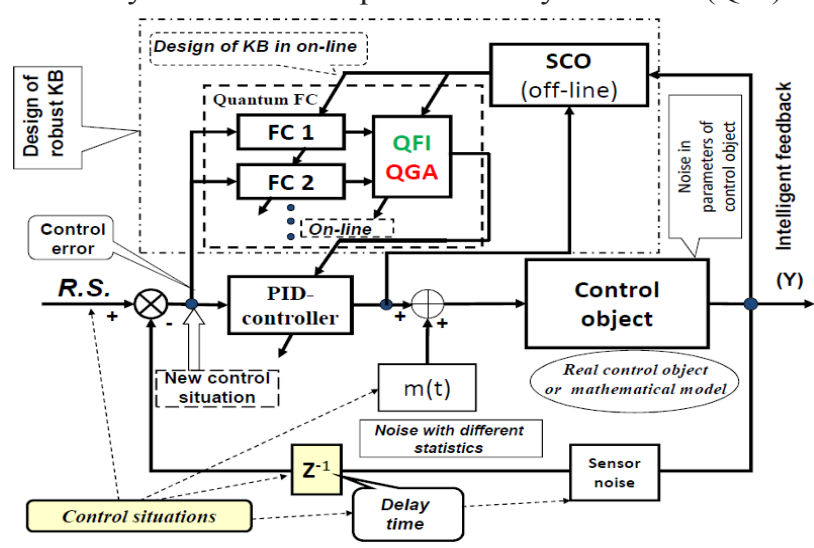

Figure 2. Structure of robust intelligent control system based on QFI

In Figure 2 the box $\mathrm{SCO}$ - soft computing optimizer toolkit $\left(\mathrm{SCOOptKB}^{\mathrm{TM}}\right)$ - design KBs of fuzzy controllers (FC1 and FC2) on learning situations that can be non-robust (imperfect) in other control situations. The input of the quantum fuzzy inference (Box QFI) with QGA extract a hidden quantum information from imperfect knowledge base (KB) responses of fuzzy controllers $\mathrm{FC} 1$ and $\mathrm{FC} 2$ on unpredicted control errors realized quantum fuzzy controller in on line without changing production rules numbers of $\mathrm{KBs}$ in $\mathrm{FC} 1$ and $\mathrm{FC} 2^{[1]}$.

\subsubsection{QFI Structure}

It is shown that in quantum computing the construction of a universal quantum simulator based on classical effective simulation is possible ${ }^{[1-7]}$. In the general form, the model of quantum algorithm computing comprises the following five stages:

- preparation of the initial state $\left|\psi_{\text {out }}\right\rangle$ (classical or quantum);

- execution of the Hadamard transform for the initial state in order to prepare the superposition state;

- application of the entangled operator or the quantum correlation operator (quantum oracle) to the superposition state;

- application of the interference operator;

- application of the measurement operator to the result of quantum computing $\left|\psi_{\text {out }}\right\rangle$.

Hence, a quantum gate approach can be used in a global optimization of KB structures of ICSs that are based on quantum computing, on a quantum genetic search and quantum deep learning algorithms ${ }^{[5,7]}$.

\subsubsection{Quantum Information Resources in QFI Al- gorithm}

Figure 3 shows the algorithm for coding, searching and 
extracting the value information from two KBs of fuzzy PID controllers designed by SCO.

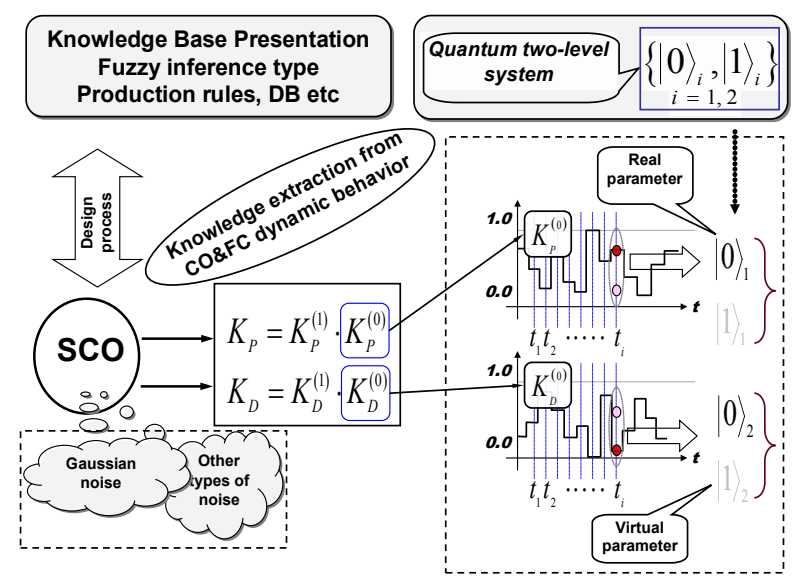

Figure 3. Example of information extraction in QFI

Thus, the quantum algorithm for QFI (Figure5) the following actions are realized ${ }^{[1,2]}$ :

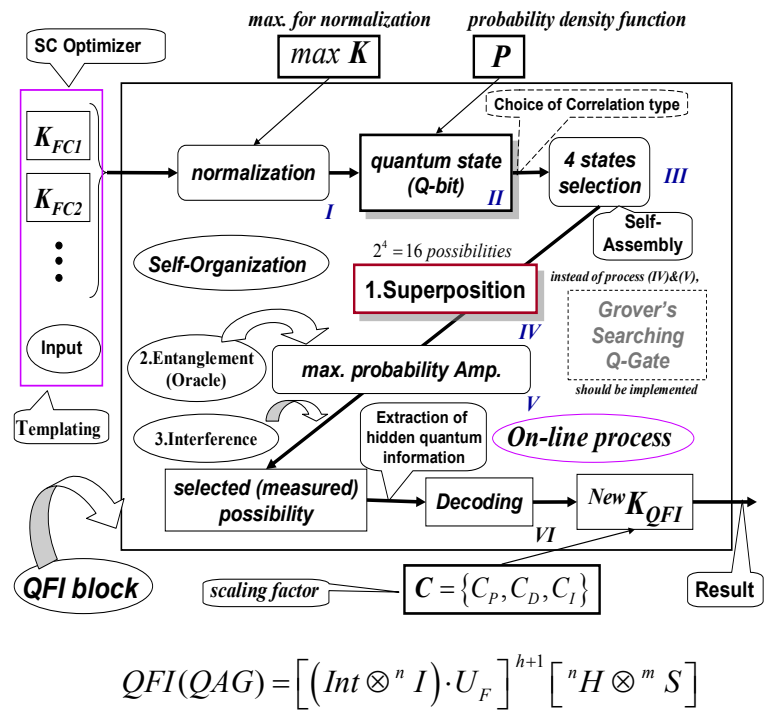

Figure 4. The structure of QFI gate

- The results of fuzzy inference are processed for each independent FC;

- Based on the methods of quantum information theory, valuable quantum information hidden in independent (individual) knowledge bases is extracted;

- In on-line, the generalized output robust control signal is designed in all sets of knowledge bases of the fuzzy controller.

- In this case, the output signal of QFI in on-line is an optimal signal of control of the variation of the gains of the PID controller, which involves the necessary (best) qualitative characteristics of the output control signals of each of the fuzzy controllers, thus implementing the self-organization principle. 


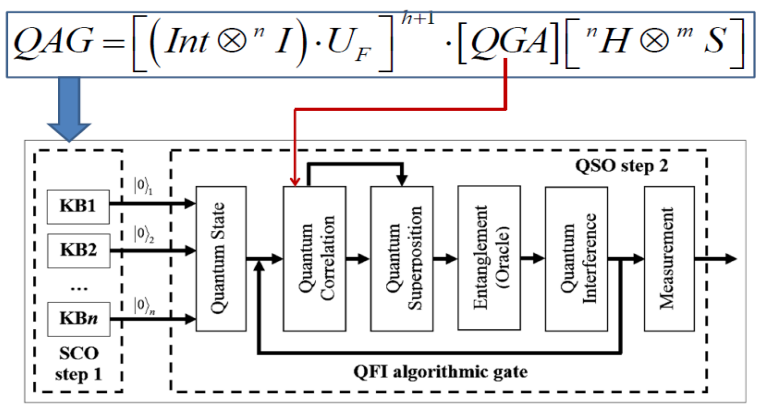

Figure 5. QAG structure of QFI with QGA

In present Part I of this article we discuss the physical models of control objects and algorithmic peculiarities of toolkit for the design the final state of quantum self-organization algorithm on Figure1 as the control goal - support of abovementioned information-thermodynamic trade-off between main measures of control qualities of intelligent cognitive control applying imperfect KB's of FCs.

Applications and sophisticated computational intelligence quantum software toolkit are discussed in Part II.

\section{Models of Control Objects (Port-controlled Hamiltonian Systems)}

Port-controlled Hamiltonian ( $\mathrm{PCH}$ ) systems are the generalization of conventional Hamiltonian systems. They can describe not only mechanical systems but also a broad class of physical systems including passive electro-mechanical systems, hydraulic, gas, mechanical systems with non-holonomic constraints and their combinations ${ }^{[14,15]}$. We briefly describe the main idea of PCH systems model.

\subsection{Port-controlled Hamiltonian Models}

A novel energy dissipating hybrid control framework was developed for Euler-Lagrangian, port-controlled Hamiltonian systems (PCHS) ${ }^{[15]}$, and lossless dynamical systems. A time-varying PCHS with dissipation is a system of the form:

$$
\dot{x}=[J(x, t)-R(x, t)] \frac{\partial H(x, t)^{\mathrm{T}}}{\partial x}+g(x, t) u, \quad y=g(x, t)^{\mathrm{T}} \frac{\partial H(x, t)^{\mathrm{T}}}{\partial x}
$$

with a Hamiltonian $H(x, t) \in \mathbb{R}, u, y \in \mathbb{R}^{m}, x \in \mathbb{R}^{n}$, a skew symmetric matrix $J(x, t)$, i.e. $J=-J^{\mathrm{T}}$ holds, and a positive semi-definite symmetric matrix $R(x, t) \geq 0$ are the interconnection and damping matrices, respectively. A PCHS is passive ${ }^{[14]}$ if

$\int_{0}^{t} u^{\mathrm{T}} y d t=H(x, t)-H(x, 0)+\int_{0}^{t}\left(\frac{\partial H(x, t)}{\partial x}\right)^{\mathrm{T}} R(x, t) \frac{\partial H(x, t)}{\partial x} d t$

The essential fact about PCHS's is that, in a given sense, power preserving interconnection of several PCHS yields a Hamiltonian system (with or without ports). The rigorous result is that interconnection of PCHS by means of what is called a Dirac structure yields an implicit PCHS. A basic property of PCHS is the energy balancing property $\frac{d H}{d t}(x, t) \leq u^{\top}(t) y(t)$ showing that a PCHS is passive if the Hamiltonian $H$ is bounded from below. Physically the internal interconnection structure is power-conserving and the term $u^{\mathrm{T}}(t) y(t)$ is the externally supplied power.

It is well-known ${ }^{[14]}$ that physically different subsystems of complex control objects (with different physical nature of subsystems) presented as Port-controlled Hamiltonian (PCH) systems and can be considered as generalization structures of conventional Hamiltonian systems ${ }^{[15]}$. We briefly describe the main idea of PCH systems model.

\subsection{Port-controlled Hamiltonian Quantum Mod- els}

The quantum port-Hamiltonian framework is the generalization of conventional model of $\mathrm{PCH}$ and can be illustrated on its application using RLC circuits ${ }^{[16]}$. These RLC circuits are then used to construct the primitive gate set for universal quantum computation, i.e., the phase-shift, Hadamard, and CNOT gates, and are synthesized with the composite Dirac structures (see, the definition Dirac structure in item 4.1) of the quantum port-Hamiltonian framework.

Model presentation of quantum PHS. For this purpose, firstly, a Schrödinger equation for the universal RLC quantum circuit is introduced applying the definition of a quantum port-Hamiltonian system. Let $P_{1} \in \mathbb{C}^{n \times n}$ be invertible and self-adjoint, let $P_{0} \in \mathbb{C}^{n \times n}$ be skew-adjoint, i.e., $P_{0}^{\dagger}=-P_{0}$ , and let $\mathcal{H} \in L^{\infty},\left([j=0, \ldots, N] ; \mathbb{C}^{n \times n}\right)$, where $j \in \mathbb{N}$ such that $\mathcal{H}_{j}^{\dagger}=\mathcal{H}_{j}, m I \leq \mathcal{H}_{j} \leq M I$ for a.e. $j \in[0, N]$ and constants $m ; \mathrm{M}>$ 0 independent of $\mathbf{j}$. The Hilbert space $X:=L^{2}\left([j=0, \ldots, N] ; \mathbb{C}^{n}\right)$ with the discrete inner product (see the standard definition of quantum mechanics, for example ${ }^{[7]}$ )

$\langle\psi, \varphi\rangle_{X}=\frac{1}{4} \sum_{j=1}^{N-1}\left\langle\varphi_{0}\left|\mathcal{H}_{0}\right| \psi_{0}\right\rangle+\frac{1}{2} \sum_{j=1}^{N-1}\left\langle\varphi_{j}\left|\mathcal{H}_{j}\right| \psi_{j}\right\rangle+\frac{1}{4} \sum_{j=1}^{N-1}\left\langle\varphi_{N}\left|\mathcal{H}_{N}\right| \psi_{N}\right\rangle$.

Then the linear first-order Schrödinger equation $i \hbar \frac{d}{d t} \mid$ $\psi(t)\rangle=\frac{P_{1}}{2} \sum_{j}\left(\mathcal{H}_{j+1}-\mathcal{H}_{j-1}\right)\left|\psi_{j}(t)\right\rangle+\frac{P_{1}}{2} \sum_{j} \mathcal{H}_{j}\left(\left|\psi_{j+1}(t)\right\rangle-\left|\psi_{j-1}(t)\right\rangle\right)+$

$P_{0} \sum_{j} \mathcal{H}_{j}\left|\psi_{j}(t)\right\rangle$ is a quantum PHS $\mathrm{H}_{j}$, where $\hbar$ is the redu ced Planck constant, the imaginary number $i=\sqrt{-1}$, and the instantaneous state of the quantum system is $|\psi(t)\rangle=$ $\sum_{j} \alpha_{j}(t)|j\rangle$, where the complex number $\alpha_{j}(t)$ is $\alpha_{j}(t)=$ $\langle j \mid \psi(t)\rangle$ and $\left\langle j^{\prime} \mid j\right\rangle=\delta_{i j^{\prime}}$. 
Example. Let us consider a state space manifold $\chi$ and a Hamiltonian $H: \chi \rightarrow \mathbb{C}$, defining energy-storage, e.g. current $I$ or voltage $V$. A quantum port-Hamiltonian system on $\chi$ is defined by the Dirac structure $\mathcal{D} \subset \mathcal{T}_{\chi} \chi \otimes \mathcal{T}_{\chi}^{\dagger} \chi \otimes \mathcal{F}_{p} \otimes \mathcal{E}_{p}$ having energy-storing port $\left(f_{\mathcal{S}}, e_{\mathcal{S}}\right) \in \mathcal{T}_{\chi} \chi \otimes \mathcal{T}_{\chi}^{\dagger} \chi$, and an external structure $\mathrm{P}$, e.g. source voltage or output current, such that $\mathcal{P} \subset \mathcal{F}_{p} \otimes \mathcal{E}_{p}$, corresponding to an external port $\left(f_{p}, e_{p}\right) \in \mathcal{F}_{p} \otimes \mathcal{E}_{p}$. The temporal dynamics of the quantum system are then specified by $\left\{\dot{\alpha}_{j}(t)=\frac{\partial}{\partial \pi_{j}}\langle H\rangle,-\dot{\pi}_{j}=\frac{\partial}{\partial \alpha_{j}}\langle H\rangle, f_{\mathcal{P}}(t), e_{\mathcal{p}}(t)\right\} \in \mathcal{D}\left(\alpha_{j}(t)\right), \quad t \in \mathbb{R}$, where $\alpha_{j}$ are the generalized coordinates, and $\pi_{j}$ are the conjugate momenta.

For purposes of quantum state preparation with universal RLC circuit design adapting some classical theory of the Operational Transconductance Amplifier (OTA) ${ }^{[16]}$ as seen in Figure 6.

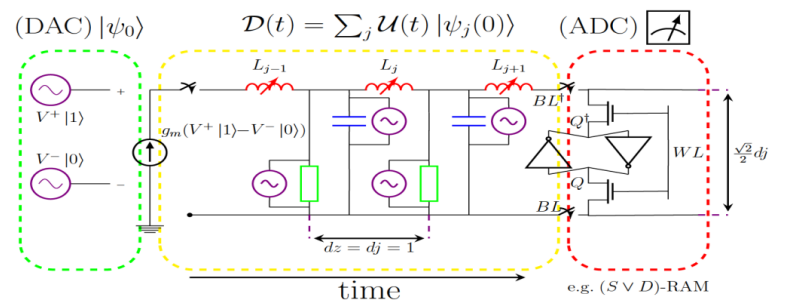

(a) RLC Quantum Channel $-\left\langle e_{V} \mid f_{V}\right\rangle=\left\langle f_{I} \mid e_{I}\right\rangle$

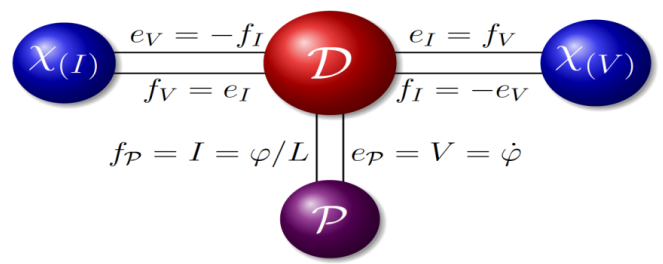

$\left\langle e_{V} \mid f_{V}\right\rangle+\left\langle e_{I} \mid f_{I}\right\rangle+\left\langle e_{\mathcal{P}} \mid f_{\mathcal{P}}\right\rangle=0 \mapsto \dot{H} \leq\left\langle e_{\mathcal{P}} \mid f_{\mathcal{P}}\right\rangle$

(b) Quantum Port-Hamiltonian System

Figure 6. (a) RLC circuit equivalent of the quantum channel.

(b) Quantum Port-Hamiltonian System representation of the RLC quantum channel (a)

Let us describe the details on Figure 6. On Figure 6a Digital-to-Analog Conversion (DAC) is used to prepare the analog quantum state, which then propagates along the $z$-direction. The time-dependent Dirac structure $\mathcal{D}(t)$ then performs a unitary evolution of the prepared quantum state. The current across the resistor(s) is $I_{R}(t)=\left|I_{j}\right| \sin \left(\varphi_{j}\right)$, the current across the capacitor(s) is $I_{C}(t)=i \omega_{j} C_{j}\left|V_{j}\right| \cos \left(\varphi_{j}\right)$, and $\varphi_{j}(t)=\omega_{j} t$ is the RLC quantum phase. The output of the Dirac structure then undergoes Analog-to-Digital Conversion (ADC), such that the quantum information is stored (cf. measurement) in
Random-Access Memory (RAM) cell(s). The port interconnection of current $I$ and voltage $V$, the external port $\mathrm{P}$, where $\chi(I)$ is the current storage state, $\chi(V)$ is the voltage storage state, the Dirac structure D links the storage ports (flows $\left\{f_{I}, f_{V}\right\}$ and efforts $\left\{e_{I}, e_{V}\right\}$; diagram is shown on Figure 6,b, respectively), with the external port (flow $f_{\mathcal{P}}$ and effort $e_{\mathcal{P}}$ ). The electric power-balance (conservation) equation is $\dot{H}=i \hbar \partial_{t t}$, such that the total power is equal to zero (color online).

The output current of an OTA is proportional to the input signal voltage, such that $I_{\text {out }}=g_{m} .\left(V_{\text {in }}^{+}(t)-V_{\text {in }}^{-}(t)\right)$ and the $j$-th RLC circuit site current with continuous (time-dependent) phases [10] is described as

$\left|I_{j}^{\text {out }}(t)\right|=\frac{g_{m}}{\exp \left\{i \omega_{I} t-\phi\right\}}\left[\left|V_{j}^{+}\right| \exp \left\{i \omega_{j}^{+} t\right\}|1\rangle-\left|V_{j}^{-}\right| \exp \left\{i \omega_{j}^{-} t\right\}|0\rangle\right]$.

For clarification purposes cf. qubits, here it should be pointed out that the quantum superposition state(s) prepared by the OTA(s) is $|\psi(t)\rangle=\left|I_{j}^{\text {out }}(t)\right\rangle$, such that $|\psi(t)\rangle_{+}=\sqrt{I_{j}^{+}} \exp \left\{i \omega_{I}^{+} t-\phi^{+}\right\}|1\rangle, \quad|\psi(t)\rangle_{-}=\sqrt{I_{j}^{-}} \exp \left\{i \omega_{I}^{-} t-\phi^{-}\right\}|1\rangle ;$

and the superposition state $|\psi(t)\rangle=|\psi(t)\rangle_{+} \pm|\psi(t)\rangle_{-}$ . Hence, from Ohm's law, the output voltage amplifier has prepared for ADC the OTA output voltage state equation $V_{\text {out }}(t)=R_{L} I_{j}^{\text {out }}(t)$. Finally, it can obtain the coupled Schrödinger equations governing the electrodynamics of the RLC quantum channel

$$
\begin{aligned}
& i \hbar \dot{V}_{j}(t)|\psi(t)\rangle_{+}=\frac{1}{2 m}\left[I_{j-1}(t)-I_{j+1}(t)\right]|\psi(t)\rangle_{+}-\left|I_{j}\right| \sin \left(\frac{2 \pi}{\varphi_{0}} \varphi_{j}(t)\right)|\psi(t)\rangle_{-}, \\
& i \hbar \dot{V}_{j}(t)|\psi(t)\rangle_{-}=\frac{1}{2 m}\left[I_{j-1}(t)-I_{j+1}(t)\right]|\psi(t)\rangle_{-}-\left|I_{j}\right| \sin \left(\frac{2 \pi}{\varphi_{0}} \varphi_{j}(t)\right)|\psi(t)\rangle_{+} ;
\end{aligned}
$$

where $I_{j}(t)$ is the current, $V_{j}(t)$ is the voltage, $\varphi_{j}(t)$ is the RLC quantum phase, i.e.

$$
\begin{aligned}
& \dot{I}_{j}(t)=-\frac{1}{2 L_{j}}\left[V_{j+1}(t)-V_{j-1}(t)\right], \\
& \dot{V}_{j}(t)=-\frac{1}{2 C_{j}}\left[I_{j+1}(t)-I_{j-1}(t)+2\left|I_{j}\right| \sin \left(\frac{2 \pi}{\varphi_{0}} \varphi_{j}(t)\right)\right], \dot{\varphi}_{j}(t)=V_{j}(t) .
\end{aligned}
$$

The solution of Equation (6) is a bright soliton propagation along the RLC quantum channel as seen in Figure $6 \mathrm{a}$. As such, the analytical solution is ${ }^{[16]}$

$$
\begin{gathered}
\varphi_{j}(t)=2 \frac{\varphi_{0}}{\pi} \tan \left[\exp \left\{\frac{j-v t}{\lambda \sqrt{1-\frac{v^{2}}{c^{2}}}}\right\}\right], \quad V_{j}(t)=\frac{\varphi_{0}}{2} \dot{\varphi}_{j}(t)=-\frac{\varphi_{0} \omega}{2 \pi} \frac{2 v}{\sqrt{1-\frac{v^{2}}{c^{2}}}} \operatorname{sech}\left[\frac{j-v t}{\lambda \sqrt{1-\frac{v^{2}}{c^{2}}}}\right] \\
I_{j}(t)=-\frac{\varphi_{0}}{4 \pi L_{j}}\left(\varphi_{j+1}(t)-\varphi_{j-1}(t)\right)=-\frac{\varphi_{0}}{2 \pi L_{j} \lambda} \frac{2}{\sqrt{1-\frac{v^{2}}{c^{2}}}} \operatorname{sech}\left[\frac{j-v t}{\lambda \sqrt{1-\frac{v^{2}}{c^{2}}}}\right]
\end{gathered}
$$

The statement of the Heisenberg uncertainty principle is the following: $\left\langle\hat{x}^{2}\right\rangle\left\langle\hat{p}^{2}\right\rangle \geq \frac{1}{4} \hbar^{2}$.

Furthermore, here it should be pointed out that the position observable $\hat{x}=z=j \Delta z=j$, and the momentum ob- 
servable $\hat{p}=-i \hbar \partial^{1 / 2} / \partial z^{1 / 2}$, thereby satisfying the Heisenberg uncertainty principle $\left\langle\hat{x}^{2}\right\rangle\left\langle\hat{p}^{2}\right\rangle \geq \frac{1}{4} \hbar^{2}$.

\subsection{Information-thermodynamic Laws of Intelligent Cognitive Control}

Let us describe control object model as (4) in the generalized form

$$
\frac{d q}{d t}=\varphi(q, S(t), t, u, \xi(t)), u=f\left(q, q_{d}, t\right),
$$

where $q$ is the vector of generalized coordinates describing the dynamics of the controlled plant; $S$ is the generalized entropy of dynamic system (4); $u$ is the control force; $q_{d}(t)$ is reference signal, $\xi(t)$ is random disturbance.

Remark: Definitions of Stability Conditions and Instability Theorems. To describe a nonlinear system's behavior, two theorems help to characterize the essential features of its motion. In addition, by bounding the Lyapunov function between these Theorems, both necessary and sufficient conditions are a result of the transition of the time derivative of the Lyapunov function from stable to unstable ${ }^{[15]}$.

1) Lyapunov Theorem for Stability. Assume that there exists a scalar function $V$ of the state $x$, with continuous first order derivatives such that

(a) $V(x)$ is positive definite; (b) $\frac{d V}{d t}$ is negative definite; (c) $V(x) \rightarrow \infty$ as $\|x\| \rightarrow \infty$.

Then the equilibrium at the origin is globally asymptotically stable.

2) Chetaev Theorem for Instability. Considering the equations of disturbed motion, let $V$ be zero on the boundary of a region $\mathrm{R}$ which has the origin as a boundary point, and let both $V$ and $V$ be positive-definite in $R$; then the undisturbed motion is unstable at the origin.

The necessary and sufficient conditions of asymptotic stability of dynamic system (7) with $\xi(t) \equiv 0$ are determined by the physical constraints (for example, as for PCHS) on the form of the Lyapunov $V$ function:

$$
\begin{array}{ll}
\text { (I) } V>0 ; \quad \text { (II) } \frac{d V}{d t} \leq 0 .
\end{array}
$$

The generalized Lyapunov function has the form ${ }^{[6]}$ : $V=\frac{1}{2} \sum_{i=1}^{n} q_{i}^{2}+\frac{1}{2} S^{2}$,

where $S=S_{p}-S_{c}$ is the production of entropy in the open system "control object + controller"; $S_{p}=\Psi(q, \dot{q}, t)$ is the production of entropy in the controlled plant; and $S_{c}=\Upsilon(\dot{e}, t)$ is the production of entropy in the controller (actuator of the automatic control system).

Thus, taking into account (7), received final expression as following:

$$
\underbrace{\frac{d V}{d t}}_{\text {Stability }}=\underbrace{\sum_{i} q_{i} \varphi_{i}(q,(\Psi-\Upsilon), t, u)}_{\text {Controllability }}+\underbrace{(\Psi-\Upsilon)(\dot{\Psi}-\dot{\Upsilon})}_{\text {Robustness }} \leq 0
$$

Remark. Let us briefly consider Equation (8). Derivative of the function $V$ can be described as $\frac{d V}{d t}=\sum_{i=1}^{n} q_{i} \dot{q}_{i}+S \dot{S}$ $=\sum_{i=1}^{n} q_{i} \varphi(q, S(t), t, u, \xi(t))+(\Psi-\Upsilon)(\dot{\Psi}-\dot{\Upsilon})$ and we have Equation (9). The function $V$ should be satisfied to conditions (I) and (II).

Relation (9) displayed stability, controllability, and robustness trade-off properties.

Remark. Equation (9) is an analytic form of the interrelations between control qualities with entropy definitions as the measure of loss controllability and robustness. This condition describes the possibility for optimum searching distribution of control level qualities for guaranteed control goal achievement in unpredicted control situations with minimal loss of working resource applying as fitness function of genetic algorithms generalized entropy from r.h.s. in (9).

Example: Information interrelations of control qualities. For (9) apply the well-known interrelation between Gibbs thermodynamic and Shannon information entropies. The Shannon entropy $H(X ; s)$ agrees with the usual thermodynamical entropy $S=\left(1-\beta \frac{\partial}{\partial \beta}\right) \log Z(\beta)$ for a physical system with Hamiltonian $\mathrm{H}$ and partial function $Z(\beta)$ at inverse temperature $\beta=\frac{1}{T}>0$, and free energy $F=-\log Z(\beta)$. For the density probability function $P(n)=\frac{e^{-\beta \eta_{n}}}{Z(\beta)}$ and $Z(\beta)=\operatorname{Tr}\left(e^{-\beta \mathrm{H}}\right), \operatorname{Spec}(\mathrm{H})=\lambda_{n}$ the expression of Shannon information entropy $[17,18]$ is as following:

$$
\begin{aligned}
H=-\sum_{n} P(n) \log P(n) & =\sum_{n} P(n) \log Z(\beta)-\beta \sum_{n} P(n) \lambda_{n} \\
& =\left(\left[\sum_{n} P(n)=1\right] \log Z(\beta)-\left[\sum_{n} P(n) \lambda_{n}=\frac{\partial}{\partial \beta} \log Z(\beta)\right]\right), \\
& =\left(1-\frac{\partial}{\partial \beta}\right) \log Z(\beta)
\end{aligned}
$$

where $\sum_{n} P(n) \lambda_{n}=\frac{\partial}{\partial \beta} \log Z(\beta)$.

Thus, both quantitative measures of the entropy

$S=\left(1-\beta \frac{\partial}{\partial \beta}\right) \log Z(\beta)=-\sum_{n} P(n) \log P(n)=H$ are equivalent $^{[18]}$.

Therefore, the entropies $S$ and $H$ connected ${ }^{[19,20]}$ as following: $S=k H=-k \sum p_{i} \ln p_{i}$, where $k \approx 1.38 \times 10^{-23}$ дж/K is Boltzmann constant. We can introduce in (6) this relation for $S(t)$ as Shannon information entropy $H$. As result have following interrelation: 
$\underbrace{\frac{d V}{d t}}_{\text {stability }}=\underbrace{\sum_{i=1}^{n} q_{i} \cdot \varphi\left(q, t, k\left(H_{c o}-H_{\text {contr }}\right), u\right)}_{\text {controlability }}+k \underbrace{\left(H_{c o}-H_{\text {contr }}\right) \cdot\left(\dot{H}_{c o}-\dot{H}_{\text {contr }}\right)}_{\text {robustmess }} \leq 0$

Therefore, Equation (10) also connect control qualities but on information entropy amount platform. That means it possible design information-thermodynamic control force ${ }^{[9]}$ in unpredicted control situations with minimum information amount on environments and CO-states.

Therefore, Equation (9) and (10) are the mutual equation system that solution defines the control force for guaranteed achievement of control goal in unpredicted control situations with minimal loss of value working resource and minimal required initial information.

Example: Information-thermodynamic analysis of cognitive control. The generalization of Equation (9) and (10) has the form:

$$
\begin{aligned}
& \underbrace{\frac{d V}{d t}}_{\text {stability }}=\underbrace{\sum_{i=1}^{n} q_{i} \cdot \varphi\left(q, t, k\left(S_{c o}-\left(S_{T C}+S_{C c}\right)\right), u\right)}_{\text {controllability }}+\underbrace{\left(S_{C o}-\left(S_{T C}+S_{C C}\right)\right) \cdot\left(\dot{S}_{C o}-\left(\dot{S}_{T c}+\dot{S}_{C c}\right)\right)}_{\text {robustess }} \leq 0,
\end{aligned}
$$

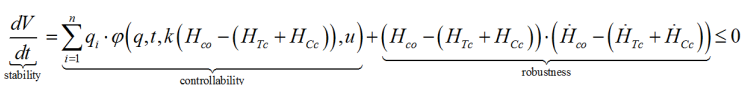

where $\left(S_{T c}+S_{C c}\right)$ and $\left(H_{T c}+H_{C c}\right)$ means the common thermodynamic and information entropies of technical and cognitive controllers correspondently.

Under control with information feedback [21,22] the extracted work $W$ defined as

$$
W \leq k T I_{C} \text {, }
$$

where $I_{c}$ is the so-called transfer entropy from the system to the measurement ${ }^{[21-23]}$.

An explicit finite-time counterpart to the equation for $W$ characterize the maximum work extractable using feedback control, in terms of the transfer entropy. The maximum amount of extractable work over $t, W_{\max }(t)$, has ${ }^{[23]}$ the following definition:

$$
W_{\max }(t)=k \int_{0}^{t} T_{\min } \dot{I}_{C} d t^{\prime} \leq k T I_{C}(t)
$$

It was shown $[22,23]$ that

$$
W_{\max }(t) \simeq k \int_{0}^{t} T_{\min } \dot{I}_{C} d t^{\prime},
$$

and $W \leq k T I_{C}(t)$ thus the second law is validated.

Example. The considered dynamic system ${ }^{[23]}$ with a feedback controller (the demon) with access to noisy voltage measurements, shown in Figure 7.

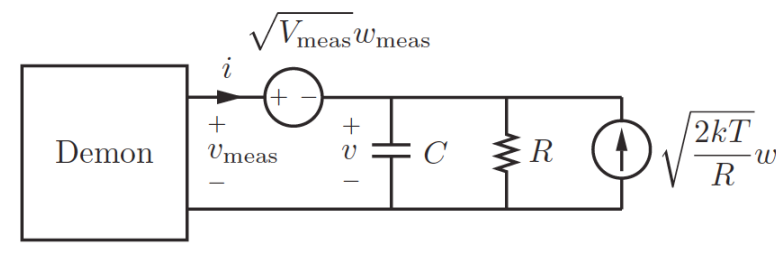

Figure 7. The demon (the feedback controller) connected to a capacitor
The demon (see a definition of Demon in ${ }^{[9]}$ ) may choose the current $\mathrm{i}$ freely and has access to the noisy voltage measurement $\vartheta_{\text {meas }}$. Consider the overdamped Langevin equation ${ }^{[23]}$

$\tau \dot{\vartheta}==-\vartheta+R i+\sqrt{2 k T R} w, \quad \tau=R C,\langle\vartheta(0)\rangle=0 ; \quad \vartheta_{\text {meas }}=\vartheta$

$+\sqrt{V_{\text {meas }}} w_{\text {meas }}$,

with $\vartheta(0)$ Gaussian, w and $\mathrm{w}_{\text {meas }}$ uncorrelated Gaussian white noise $\left(\left\langle w(t) w\left(t^{\prime}\right)\right\rangle=\left\langle w_{\text {meas }}(t) w_{\text {meas }}\left(t^{\prime}\right)\right\rangle=\delta\left(t-t^{\prime}\right)\right) ; V_{\text {meas }}$ the intensity of the measurement noise. The work-extraction rate of the demon is $\dot{W}$, can define that, $\dot{U}=\dot{Q}-\dot{W}$, where $U=\frac{1}{2} C\left(\vartheta^{2}\right) \equiv \frac{1}{2} k T_{C}, \dot{Q}=\frac{k}{\tau}\left(T-T_{C}\right) . \dot{W}=-\left\langle\vartheta_{i}\right\rangle$.

The voltage $\vartheta_{\text {meas }}$ is the measurement that supplies the demon with information. The Kalman-Bucy filter state is denoted $\hat{\vartheta}$ and we have: $\tau \frac{d}{d t} \hat{\vartheta}=-\hat{\vartheta}+R i+K\left(\vartheta_{\text {meas }}-\hat{\vartheta}\right), \quad \hat{\vartheta}(0)=0$ , where $K$ is an optimal gain $(K)_{0}^{t}$, the Kalman gain, that will minimize the estimation error variance $\min _{(\kappa)_{0}^{\prime}}\left\langle[\vartheta(t)-\hat{\vartheta}(t)]^{2}\right\rangle$ (a filter Riccati equation, for example ${ }^{[23]}$ ) is

$\tau \dot{T}_{\min }=2\left(T-T_{\min }\right)-\frac{\sigma T_{\min }^{2}}{2 T}, \quad T_{\min }(0)=T$,

where $K=\frac{\sigma T_{\text {min }}}{2 T}, \quad \sigma \equiv \frac{2 k T R}{V_{\text {mas }}}$ and maximum work amount (15) quantified through the definition of the transfer entropy

[23]:

$I_{C}(t)=I\left(\left(\vartheta(0),(w)_{0}^{t}\right) ;\left(\vartheta_{\text {meas }}\right)_{0}^{t}\right)$.

or

$I_{C}(t)=\frac{1}{2 V_{\text {meas }}} \int_{0}^{t}\left\langle[\vartheta-\hat{\vartheta}]^{2}\right\rangle d t^{\prime}=\frac{1}{2 V_{\text {meas }}} \int_{0}^{t} \frac{k T_{\min }}{C} d t^{\prime}=\frac{\sigma}{4 \tau} \int_{0}^{t} \frac{T_{\min }}{T} d t^{\prime}$.

Equation (11) shows that the level of robustness possible to increase apply entropy rate production of technical controller that decrease the loss of value resource of control object. Equation (12) shows that the negentropy of cognitive controller decrease the requirements to necessary initial information amount for the achievement designed level of robustness. Thus, the extracted information from knowledge base of cognitive controller gives the possibility to receive the additional resource for value work that equivalence to purposeful action on control object for quarantined achievement of control goal. That means the information is physical and the control action based on this information can produce the value work.

Figure 8 shows (see Equation (11), (12) and (14)) the information-physical law for design of smart control. 


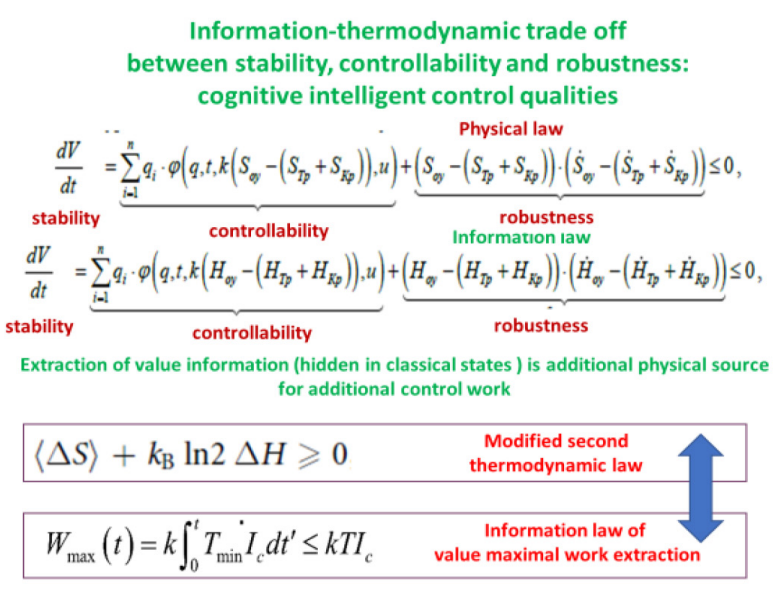

Figure 8. Information-thermodynamic trade-off between control qualities

Let us consider these statements applying analysis of the interrelation between information amount, extracted value work and free energy, and for (5) the following relation is defined:

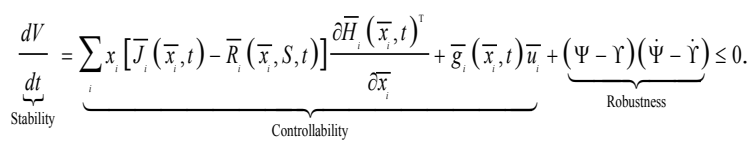

The state corresponds to the minimum system entropy and called as "equipartition of energy" ${ }^{[24]}$

Remark. According to (15) it is possible define

$\sum q_{i} \varphi_{i}(q,(\Psi-\Upsilon), t, u) \leq(\Psi-\Upsilon)(\dot{\Upsilon}-\dot{\Psi})$.

And from (19):

(1) $\sum q_{i} \dot{q}_{i}<0,(\Psi>\Upsilon),(\dot{\Upsilon}>\dot{\Psi}), S \dot{S}>0$;

(2) $\sum q_{i} \dot{q}_{i}<0,(\Psi<\Upsilon),(\dot{\Upsilon}<\dot{\Psi}), S \dot{S}>0$

(3) $\sum q_{i} \dot{q}_{i}<0,(\Psi<\Upsilon) ;(\dot{\Upsilon}>\dot{\Psi}), S \dot{S}<0, \sum q_{i} \dot{q}_{i}<S \dot{S}$

etc. and its combinations, that means thermodynamically stabilizing compensator can constructed.

\subsection{Micro-nano-robotics Intelligent Control}

In this item the control modeling methodology of mobile micro robot in a blood flow fluid ${ }^{[25]}$ briefly described.

Entropy Production and Dynamic Stability of a Micro-Robot in a Fluid.

The micro-robot as a mechanical system in a fluid ${ }^{[25]}$ considered as:

$$
\begin{aligned}
& m_{1} \ddot{x}_{1}+C_{d} \frac{\rho}{2} A_{1}\left|\dot{x}_{1}\right| \dot{x}_{1}+K_{1}\left(x_{1}-x_{0}-l_{1} \theta_{0}\right)-K_{2}\left(x_{2}-x_{1}-l_{2} \theta_{1}\right)=\tau(t) \\
& +\xi(t), m_{2} \ddot{x}_{2}+C_{d} \frac{\rho}{2} A_{2}\left|\dot{x}_{2}\right| \dot{x}_{2}+K_{1}\left(x_{2}-x_{1}-l_{2} \theta_{1}\right)-K_{3}\left(x_{3}-x_{2}\right. \\
& \left.-l_{3} \theta_{2}\right)=0 m_{3} \ddot{x}_{3}+C_{d} \frac{\rho}{2} A_{3}\left|\dot{x}_{3}\right| \dot{x}_{3}+K_{3}\left(x_{3}-x_{2}-l_{3} \theta_{2}\right)=0(20)
\end{aligned}
$$

where $\theta_{n+1}=-\frac{1}{2} \theta_{n}+\frac{3}{2} \frac{1}{l_{n+1}}\left(x_{n+1}-x_{n}\right)$ and $m_{1}=1.6 \times 10^{-7} \mathrm{~kg} ; m_{2}$

$=1.4 \times 10^{-6} \mathrm{~kg} ; m_{3}=2.4 \times 10^{-6} \mathrm{~kg} ; l_{1}=2.0 \times 10^{-3} \mathrm{~m} ; l_{2}=4.0 \times 10$

${ }^{-3} \mathrm{~m} ; l_{3}=4.0 \times 10^{-3} \mathrm{~m} ; K_{1}=61.1 \mathrm{~N} / \mathrm{m} ; K_{2}=13.7 \mathrm{~N} / \mathrm{m} ; K_{3}=23.5$

$N / m ; A_{1}=4.0 \times 10^{-6} \mathrm{~m}^{2} ; A_{2}=2.4 \times 10^{-5} \mathrm{~m}^{2} ; A_{3}=4.0 \times 10^{-5} \mathrm{~m}^{2} ;$

$C_{d}=1.12 ; \rho=1000 \mathrm{~kg} / \mathrm{m}^{3}$;

$\xi(t)$ - stochastic excitation (white noise).

An entropy production of MMR is defined based on Equation (20) as

$\frac{d S_{i}}{d t}=\sum_{n=1}^{3} C_{d} \frac{\rho}{2} A_{n}\left|\dot{x}_{n}\right| \dot{x}_{n}^{2}, \quad i=1,2,3$,

and the Lyapunov function defined ${ }^{[26]}$

$V=\sum_{i=1}^{3} m_{i} \frac{\dot{x}_{i}^{2}}{2}+\sum_{i=1}^{3} \frac{K_{i}\left(x_{i}-x_{i-1}-l_{i} \theta_{i-1}\right)^{2}}{2}+\frac{S^{2}}{2}$,

where $S=S_{i}-S_{c}$ and $S_{c}$ is the entropy of controller with torque $\tau$ in Equation (20).

For the traditional form representation of (20) as $\dot{x}_{i}=\varphi\left(x_{i}, \tau, t\right)$ we received ${ }^{[27]}$

$\frac{d V}{d t}=\sum_{i} \varphi_{i}\left(x_{i}, \tau, t\right) x_{i}+\left(S_{i}-S_{c}\right)\left(\frac{d S_{i}}{d t}-\frac{d S_{c}}{d t}\right)<0$

From Equation (23) sufficient conditions can be described as following:

$\sum_{i} \varphi_{i}\left(x_{i}, \tau, t\right) x_{i}<\left(S_{i}-S_{c}\right)\left(\frac{d S c}{d t}-\frac{d S_{i}}{d t}\right), \frac{d S_{c}}{d t}>\frac{d S_{i}}{d t}$.

Thus, the requirements of stability design achieved with "negentropy" amount of intelligent controller.

Figure 9 shows simulation results.
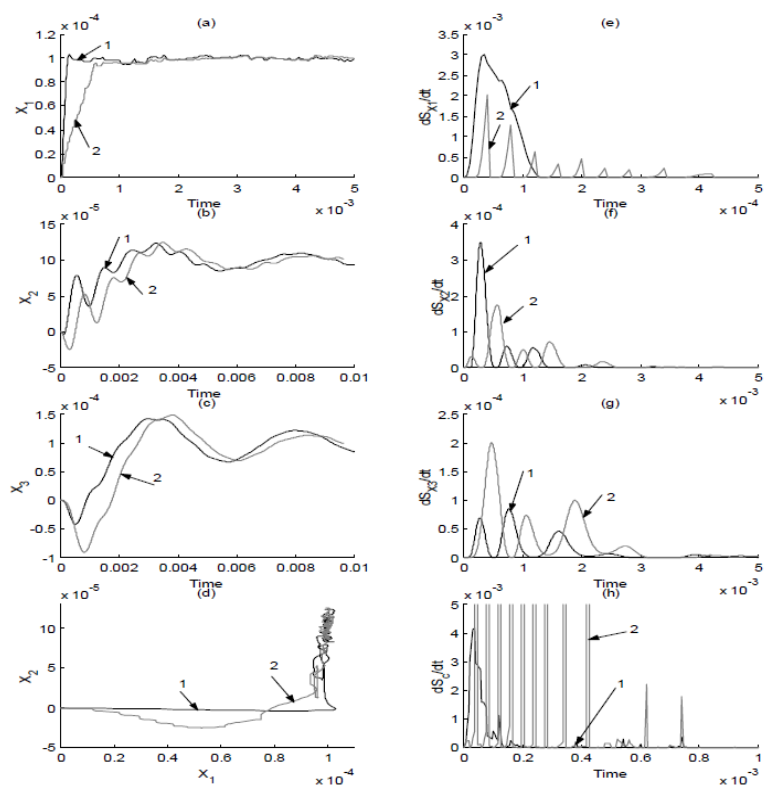

Figure 9. Simulation results of dynamic and thermodynamic behavior of MMR under different control types: 1 - GA-PID - controller with minimum of entropy production, 2 -PID controller 
Figure 10 shows the result of the intelligent behavior MNR simulation based on genetic algorithm with the fitness function as the minimum entropy production (in MNR and in conventional controller.)

Figures 9 and 10 show that the intelligent behavior of the MMR can be achieved based on computational intelligence toolkit as quantum soft computing.

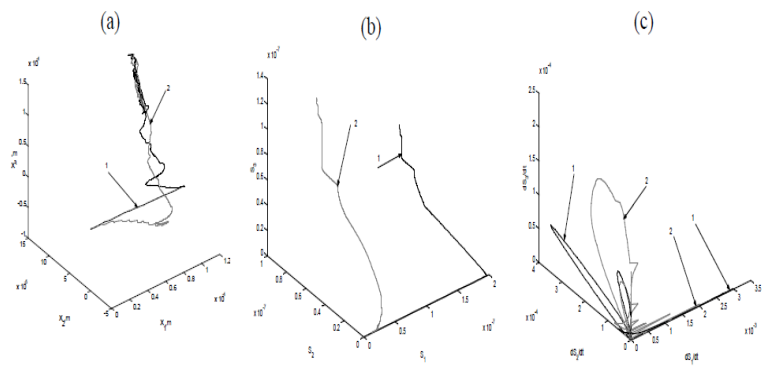

Figure 10. Simulation results of dynamic and thermodynamic behavior of MMR under different control strategies in 3D space. Curve 1 - GA-PID - controller with minimum of entropy production, curve 2 - conventional PID controller

Example. A cooperative system with entropy production and rate exchange ${ }^{[28-31]}$ can be as:

- MMR motion (on classical level):

$\ddot{\xi}+F(\xi, \dot{\xi}, t, S)+\frac{d V_{0}(\xi)}{d \xi}=u(t)$

- MMR coupled motion (non-linear Schrödinger ${ }^{[30]}$ ):

$i \hbar \frac{\partial \Psi}{\partial t}=-\frac{\hbar^{2}}{2 m} \frac{\partial^{2} \Psi}{\partial x^{2}}+\underbrace{\left\{V_{0}(x)+F(\xi, \dot{\xi}, t, S)[x-\xi(t)]\right.}_{\text {Classical motion }}+$

$\left.+\frac{\gamma \hbar}{i}(\ln \Psi-\langle\ln \Psi\rangle)\right\} \Psi-2 \chi a \frac{\partial \alpha(x, t)}{\partial x} \Psi=H_{0} \Psi$

- Fluid medium ${ }^{[31]}$

$i \hbar \frac{\partial \alpha}{\partial t}=(E+\Delta E) \alpha-R_{0} J(\omega) \frac{\partial^{2} \alpha}{\partial x^{2}}-G|\alpha|^{2} \alpha-$

$-\frac{i \hbar}{2} Z \alpha+F_{f}-\frac{2 \chi a}{M} \frac{\partial}{\partial x}|\Psi|^{2}$

Parameters $E, \Delta E, R_{0}, J(\omega) G, Z, \chi$ and $\gamma$ in ${ }^{[28]}$ are defined. For $\alpha=\exp \left\{-\frac{Z}{2} t\right\} \Psi_{1}(x, t)$ and a new simple form of equation:

$i \hbar \frac{\partial \Psi_{1}}{\partial t}=(E+\Delta E) \Psi_{1}-R_{0} J(\omega) \frac{\partial^{2} \Psi_{1}}{\partial x^{2}}-G\left|\Psi_{1}\right|^{2} \Psi_{1}$

$+F_{f}-\frac{2 \chi a}{M} \frac{\partial}{\partial x}|\Psi|^{2}$

- Entropy exchange (between a quantum fluid medium and MMR):

$S=S_{p r}-S_{e x}$,

$\frac{d S_{p r}}{d t}=\frac{1}{T} F\left(\xi, \dot{\xi}, t, S_{p r}\right) \dot{\xi}$,

$$
\frac{d S_{e x}}{d t}=\Theta\left(\xi, \dot{\xi}, t, S_{1}\right) ; S_{1}=-|\Psi|^{2} \ln |\Psi|^{2}=-\operatorname{tr}(\rho \ln \rho),
$$

where $S_{p r}$ - the entropy production of the MMR motion; $S_{e x}$ - the entropy exchange between the MMR and fluid medium; $S_{1}$ - the entropy of fluid medium (von Neumann entropy); $T$ - the temperature (constant); $m, \gamma$ - real constants.

For an initial coherent state of MMR the classical equation of motion is:

$\Psi(x, t)=\langle x \mid \Psi(0)\rangle=\frac{1}{\left[2 \pi d^{2}\right]^{\frac{1}{4}}} \exp \left\{k_{0}-\frac{x^{2}}{4 d}\right\}$

From Equation (31) local soliton as the solution

$$
\alpha(x, t)=\frac{[N \mu \exp \{-Z t\}] \exp \left[i\left(k x-\omega_{0} t\right)\right]}{\cosh \left[\left(\mu / R_{0}\right) \exp (-Z t)\left(x-x_{0}-v t\right)\right]},
$$

where $\mu(x, t)=\mu_{0} \exp (-Z t), N=\int_{-\infty}^{+\infty}|\alpha(x, t)|^{2} d x, N$ the occupation number of interacting quantum modes, $k$ wave number. The solution has the following form:

$\Psi=N \exp (R+i M), \quad \tilde{x}=x-\xi(t)$.

If we introduce the ansatz ${ }^{[25]}$

$M(t, x, \xi)=K_{x}(t)+L_{x}(t) \widetilde{x}+M_{x} \dot{\widetilde{x}}^{2}=M(\widetilde{x}, t)$,

$R(t, x, \xi)=-\left(P\left(x-\xi(t)^{2}=-P \widetilde{x}^{2}=R(\tilde{x}, t)\right.\right.$,

where $K_{x}=-\frac{1}{\hbar} \int_{0}^{t} \Phi(\xi, \dot{\xi}, \tau) d \tau-\frac{1}{2} \frac{\omega_{0}^{2}}{\Omega} t, \Phi(\xi, \dot{\xi}, t)=$

$\frac{1}{2} m \dot{\xi}^{2}(t)-\frac{1}{2} m \omega_{0}^{2} \xi^{2}(t) ; \ddot{\xi}+\gamma \dot{\xi}+F(\xi, \dot{\xi}, t)+\omega_{0}^{2} \xi=0$,

$L_{x}(t)=\frac{m}{\hbar} \dot{\xi}(t), M_{x}(t)=-\frac{m \gamma}{4 \hbar}, P=\frac{m}{2 \hbar} \Omega, \Omega=\left(\omega_{0}^{2}-\right.$

$\left.\frac{\gamma^{2}}{4}\right)^{1 / 2}$,

then the soliton-like solution ${ }^{[31,32]}$

$\Psi\left(x, t, \xi(t)=\left(\frac{m \Omega}{\pi \hbar}\right)^{\frac{1}{4}}\left(\exp \frac{i}{\hbar}\left[m \dot{\xi}(x-\xi)-\frac{1}{4} m \gamma(x-\xi)^{2}\right]\right.\right.$

$\left.\left.-\frac{m}{2 \hbar} \Omega(x-\xi)^{2} \exp \left\{\frac{i}{\hbar}\left[\int_{0}^{t} \Phi(\xi, \dot{\xi}, \tau) d \tau-\frac{1}{2} \hbar \omega_{0}\left(\frac{\omega_{0}}{\Omega}\right) t\right]\right\}\right\} \phi_{n}(x-\xi(t))\right)$

where $\phi_{n}$ satisfies

$-\frac{\hbar^{2}}{2 m} \frac{d^{2} \phi_{n}}{d y^{2}}+\frac{1}{2} m \Omega^{2} y^{2} \phi_{n}=\varepsilon_{n} \phi_{n}, \quad \varepsilon_{n}=\left[n+\frac{1}{2}\right] \hbar \Omega, \quad n=$

$1,2, \ldots, y=x-\xi(t)$.

In (25) the trajectory $\xi(t)$ is classical and a spatio-temporal fluid motion shown in Figure11, a. 

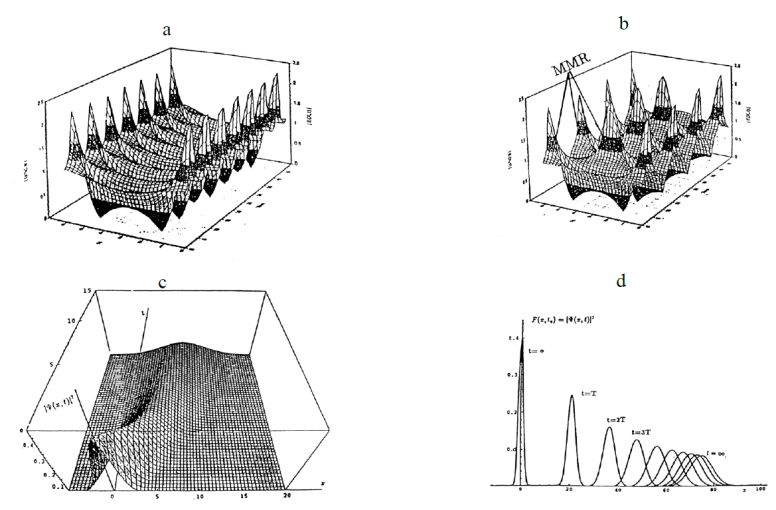

Figure 11. a) Spatio-temporal motion of fluid medium; b) Soliton like spatio-temporal motion of MNR in dissipative nonlinear medium; c) Motion wave packet in presence of dissipation; d) Projection of motion on plain $|\psi|^{2}-x$

In this case the MMR-body in the initial state located and can be coupled with the soliton inner structure (41) (see Figure 11, b,c,d) and Schrödinger-like description model equation is

$i \hbar \frac{\partial}{\partial t} \Psi(x, t)=-\frac{\hbar^{2}}{2 m} e^{-Z_{t} / m} \frac{\partial^{2}}{\partial x^{2}} \Psi(x, t)$,

and for the final state $(36)^{[30]}$

$|\Psi(x, t)|^{2}=\frac{1}{\sqrt{2 \pi\left[d^{2}+\frac{\left(t_{Z} \hbar\right)^{2}}{(2 m d)^{2}}\right]}} \exp \left[-\frac{\left(x-\frac{k_{0} t_{Z} \hbar}{m}\right)^{2}}{2\left[d^{2}+\frac{\left(t_{Z} \hbar\right)^{2}}{(2 m d)^{2}}\right]}\right]$

Here $t_{Z}=\frac{m\left(1-\exp \left(-\frac{Z_{t}}{m}\right)\right.}{Z}, d=\sqrt{\left\langle\Psi(0)\left|x^{2}\right| \Psi(0)\right\rangle}$.

$d=\sqrt{\left\langle\Psi(0)\left|x^{2}\right| \Psi(0)\right\rangle-\langle\Psi(0)|x| \Psi(0)\rangle^{2}}$.

Heisenberg principle of uncertainty defined as

$\left\langle\Delta x^{2}\right\rangle\left\langle\Delta p^{2}\right\rangle=\frac{1}{4} \frac{\hbar^{2 \omega_{0}^{2}}}{\Omega^{2}} \geq \frac{1}{4} \hbar^{2}$.

By $\gamma \rightarrow 0$ we obtain $\lim _{\gamma \rightarrow 0}\left\langle\Delta x^{2}\right\rangle\left\langle\Delta p^{2}\right\rangle=\lim _{\gamma \rightarrow 0}$ $\frac{1}{4} \frac{\hbar^{2 \omega_{0}^{2}}}{\Omega^{2}}=\frac{1}{4} \hbar^{2}$

Thus, the control vibration of MMR creates a "fur-coat" in the form of the soliton.

Therefore, mutual cooperative interrelation effect between quantum and classical levels creates artificial (biologically inspired) man-made mobile robot.

The thermodynamic equivalent model to Equation (29), (30) is ${ }^{[33,34]}$. $\dot{\rho}=\frac{1}{i \hbar}[H, \rho]+\gamma D(\rho, S)=\frac{1}{i \hbar}[H, \rho]+\gamma(S-\langle S\rangle \mathbf{1}) \rho$,

$\frac{d S}{d t}=-\alpha \frac{d V}{d t}, \alpha=\frac{1}{T}$,

where $V$ is the Lyapunov function of (29).

Thermostat Equation (45) described as

$\dot{\rho}=\frac{1}{i \hbar}[H, \rho]+\gamma\left\{(S-\langle S\rangle \mathbf{1}) \rho-\alpha\left(\frac{1}{2}[H, \rho]_{+}-\langle H\rangle \rho\right)\right\}, \frac{d S}{d t}=-\alpha$

$\frac{d V}{d t}, \alpha=\frac{1}{T}$.

If $\frac{d S}{d t}=0$, then we obtain the result ${ }^{[33,34]}$.

From Equation (29), (33) and (34) thermodynamic MMR-stability can be described as ${ }^{[27]}$ :

$$
\begin{aligned}
& \frac{d V}{d t}=\sum_{k=1}^{2} \xi_{k}\left[F(\xi, \dot{\xi}, t, S)+\frac{d V_{0}}{d \xi}\right]+\left(S_{p r}-S_{e x}\right)\left(\frac{d S_{p r}}{d t}-\frac{d S_{e x}}{d t}\right)( \\
& \text { And } \\
& \sum_{k=1}^{2} \xi_{k}\left[F(\xi, \dot{\xi}, t, S)+\frac{d V_{0}}{d \xi}\right]<\left(S_{e x}-S_{p r}\right)\left(\frac{d S_{p r}}{d t}-\frac{d S_{e x}}{d t}\right)
\end{aligned}
$$

Thus, an unstable dynamic system can be transferred into stable state applying only entropy production (intelligent control level) as a non-mechanical thermodynamic force.

Remark: Stability Lemma for Nonlinear Systems. Based on the relationship between thermodynamic exergy and Hamiltonian systems in ${ }^{[40]}$ a fundamental stability Lemma for Hamiltonian systems formulated. The stability of Hamiltonian systems is bounded between Lyapunov and Chetaev theorems as following: Given the Lyapunov derivative as a decomposition and sum of exergy generation rate $\dot{W}$ and exergy dissipation rate $T_{0} \dot{S}_{i}$ then

$\dot{V}=\dot{W}-T_{0} \dot{S}_{i}=\sum_{j=1}^{N} Q_{j} \dot{q}_{j}-\sum_{l=1}^{M-N} Q_{l} \dot{q}_{l}$.

where $Q_{j}$ is the generalized force vector and the irreversible entropy production rate can be expressed as $\dot{S}_{i}=\sum_{k} \mathcal{F}_{k} \mathcal{X}_{k}=\frac{1}{T_{0}} \sum_{k} Q_{k} \dot{q}_{k} \geq 0$. A control law is Lyapunov Optimal if it minimizes the first time derivative of the Lyapunov function over a space of admissible controls. In general, a set of feedback gains are optimized by minimizing the regulating and/or tracking error of the feedback controller while regulating to zero and/or tracking a desired reference input. The Lyapunov function is the total error energy which for most mechanical systems is equivalent to an appropriate Hamiltonian function $\mathrm{H}$ as $V=\mathrm{H}$. Then the concept of Lyapunov Optimal ${ }^{[40]}$ follows directly from setting $\dot{W}=0$ in (49) and maximizing $T_{0} \dot{S}_{i}$ for which the time derivative of the Lyapunov function (Hamiltonian) or the modified power (work/energy) equa- 
tion is written as $\dot{V}=\dot{\mathrm{H}}=-T_{0} \dot{S}_{i}=-\sum_{j=1}^{N} Q_{j} \dot{q}_{j}=-\sum_{j=1}^{N} F_{j} \dot{R}_{j}$ which is independent of system dynamics and is a kinematic quantity that applies to any system. Note that $F_{j}$ denotes a set of forces acting on a mechanical system and $\dot{R}_{j}$ denotes the inertial linear velocity of the point where $F_{j}$ is applied. Passivity control for robotic systems follows directly from setting $\dot{W}=0$ in (49).

Remark: Information-like Lyapunov functions. Recently, in ${ }^{[56]}$ presented a rich information-like family of universal Lyapunov functions for any linear or non-linear reaction network with detailed or complex balance. Moreover, $H_{f}$ are not just Lyapunov functions but divergences: $H_{f}\left(c^{1}(t) \mid c^{2}(t)\right)$ is monotonically non-increasing function of time $t$ for any two kinetic curves $c^{1}(t)$ and $c^{2}(t)$ with the same value of $\sum_{i} c_{i}$. These new functions aimed to resolve "the mystery" about the difference between the rich family of Lyapunov functions ( $f$-divergences) for linear kinetics and a limited collection of Lyapunov functions for non-linear networks in thermodynamic conditions.

\section{KB-self-organization of FC's Based on QFI with QGA}

The kernel of the abovementioned FC design toolkit is a so-called SCO implementing advanced soft computing ideas. SCO is considered as a new flexible tool for design of optimal structure and robust KBs of FC based on a chain of genetic algorithms (GAs) with information-thermodynamic criteria for KB optimization and advanced error back-propagation algorithm for KB refinement ${ }^{[2]}$. Input to $\mathrm{SCO}$ can be some measured or simulated data (called as "teaching signal" (TS)) about the modelling system. For TS design (or for GA fitness evaluation) we are used stochastic simulation system based on the control object model. More detail description of SCO is given in ${ }^{[1,2]}$. Below we discuss the application of this algorithm in QFI structure.

\subsection{Robust FC Design Toolkit}

Figure 2 illustrates as an example the structure and main ideas of self-organized control system consisting of two FC's coupling in one QFI chain that supplies a self-organizing capability. According to described above algorithm the input to the QFI gate is considered according (1) and Figure 4 as a superposed quantum state $K_{1}(t) \otimes K_{2}(t)$, where $K_{1,2}(t)$ are the outputs from fuzzy controllers FC1 and FC2 designed by $\mathrm{SCO}$ (see, in details Figure 4) for the given control task in different control situations (for example, in the presence of different stochastic noises).

The algorithm of superposition calculation is presented in Figure 12 and described in details in ${ }^{[5,6]}$.

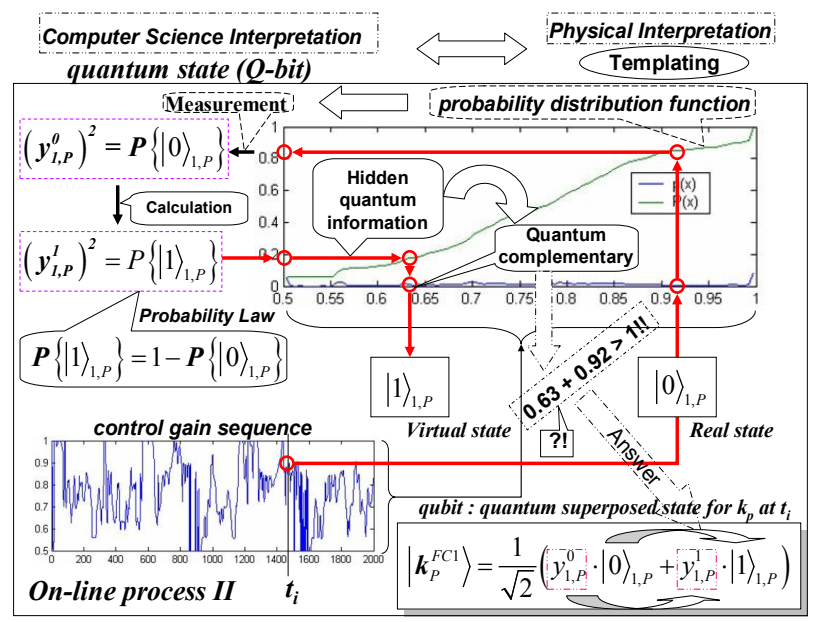

Figure 12. The algorithm of superposition calculation

We discuss for simplicity the situation in which an arbitrary amount of correlation is unlocked with a one-way communication message ${ }^{[1,2]}$. The states exhibiting this behavior need not be entangled and corresponding communication can be organized using Hadamard transform ${ }^{[5-7]}$. Therefore, using the Hadamard transformation and a new type of quantum correlation as the communication between a few KB's it is possible to increase initial information by unconventional quantum correlation (as the quantum cognitive process of a value hidden information extraction in on-line, see, e.g. Figure 5).

Let us consider a simplified case of QFI when with the Hadamard transform is organized an unlocked correlation in superposition of two KBs; instead of the difficult defined entanglement operation an equivalent quantum oracle is modelled that can estimates an "intelligent state" with the maximum of amplitude probability in corresponding superposition of classical states (minimum entropy principle relative to extracted quantum knowledge ${ }^{[5-7]}$ ).

Interference operator extracts this maximum of amplitude probability with a classical measurement.

Figure 13 shows the structure of Quantum Computing Optimizer of robust KB-FC based on QFI ${ }^{[5]}$.

Using of described QFI model to control of non-linear locally and globally unstable dynamic systems below is described. 


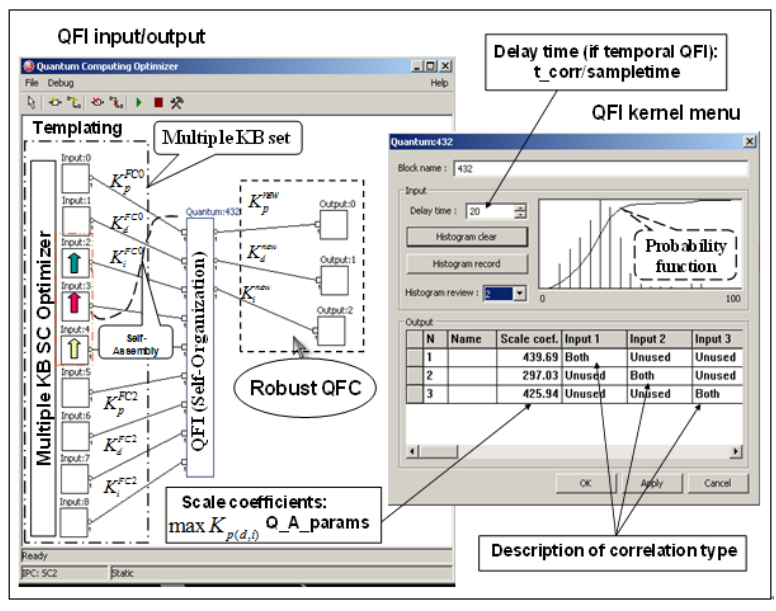

Figure 13. QFI-process by using QC Optimizer (QFI kernel)

\subsection{Benchmark's Simulation of Smart Control with QFI}

It is demonstrated that FCs prepared to maintain $\mathrm{CO}$ in the prescribed conditions are often fail to control when such a conditions are dramatically changed. We propose the solution of such kind of problems by introducing a quantum generalization of strategies in fuzzy inference in on-line from a set of pre-defined fuzzy controllers by new QFI based systems. The latter is a new quantum algorithm in quantum computation without entanglement. Two Benchmarks are considered: robust control of locally and globally unstable control objects.

\subsubsection{Benchmark 1: Globally Unstable Control Object Simulation}

"Cart - pole" control object is a non-linear dissipative system. This is a typical task of control theory, they demonstrating quality of control system. Task of control is the stability of inverted pendulum in vertical position. The motion of the dynamic system "cart - pole" is described by the following equations

$$
\begin{aligned}
& \ddot{\theta}=\frac{g \sin \theta+\cos \theta\left(\frac{u+\xi(t)+a_{1} \dot{z}+a_{3} z-m l \dot{\theta}^{2} \sin \theta}{m_{c}+m}\right)-k \dot{\theta}}{l\left(\frac{4}{3}-\frac{m \cos ^{2} \theta}{m_{c}+m}\right)} \\
& \ddot{z}=\frac{u+\xi(t)-a_{1} \dot{z}-a_{2} z+m l\left(\dot{\theta}^{2} \sin \theta-\ddot{\theta} \cos \theta\right)}{m_{c}+m},
\end{aligned}
$$

where $\theta$ is the pendulum deviation angle (degrees); $z$ is the movement of the cart $(\mathrm{m}) ; \mathrm{g}$ is the acceleration of gravity $\left(9.8 \mathrm{~m} / \mathrm{s}^{2}\right) ; m_{c}$ is the pendulum mass $(\mathrm{kg}) ; 1$ is the pendulum half-length $(m) ; \xi(t)$ is the stochastic excitation; and $u$ is the control force acting on the cart $(\mathrm{N})$. The equations for the entropy production rate in the control object and the PID controller have the following form, respectively:

$$
\frac{d}{d t} S_{\theta}=\frac{k \dot{\theta}^{2}+\frac{m l \dot{\theta}^{3} \sin 2 \theta}{m_{c}+m}}{l\left(\frac{4}{3}-\frac{m \cos ^{2} \theta}{m_{c}+m}\right)} ; \frac{d}{d t} S_{z}=a_{1} \dot{z}^{2} ; \frac{d}{d t} S_{u}=k_{d} \dot{e}^{2}
$$

The following parameter values are determined: $m_{c}=1 ; m=0.1 ; l=0.54 k=0.4 ; a_{1}=0.1 ; a_{2}=5 ;$ and the initial position $\left[\theta_{0} ; \dot{\theta}_{0} ; z_{0} ; \dot{z}_{0}\right]=[10 ; 0.1 ; 0 ; 0]$ (the value of the pendulum deviation angle is given in degrees); the constraint on the control force is $-0.5<u<5.0$.

The specific feature of control problem for the given control object (50) is the application of one fuzzy PID controller for controlling the movement of the cart (with one degree of freedom), while the control object has two degrees of freedom.

The control goal is that the pendulum deviation angle (second generalized coordinate) reaches the given value via the implicit control using the other generalized coordinate and corresponding essentially nonlinear cross-connections with the cart movement coordinate (effect of energy transmission between the generalized coordinates).

In the case of the similar initial learning conditions, the $\mathrm{SCO}$ with soft computing is used to design $\mathrm{KB} 1$ of $\mathrm{FC} 1$ for the generalized criterion of minimal mean square error:

$$
\int_{t_{0}}^{t_{\text {end }}} \theta^{2}(t) d t /\left\|\theta^{2}(t)\right\|+\int_{t_{0}}^{t_{\text {end }}} \dot{\theta}^{2}(t) d t /\left\|\dot{\theta}^{2}(t)\right\|
$$

and $\mathrm{KB}_{2}$ for $\mathrm{FC}_{2}$ for the generalized criterion of minimal absolute error of the pendulum position:

$$
\int_{t_{0}}^{t_{\text {end }}}|\theta(\tau)| d \tau /\|\theta(\tau)\|+\int_{t_{0}}^{t_{\text {end }}}|\dot{\theta}(\tau)| d \tau /\|\dot{\theta}(\tau)\| .
$$

Thus, we consider the solution of the vector (multi-objective) optimization problem based on the decomposition of the KB. The Gaussian noise was used as the random signal for designing $\mathrm{KB}_{1}$, and Rayleigh noise was used for forming $\mathrm{KB}_{2}$ (see Figure 14, learning situations (S1, S2), respectively).

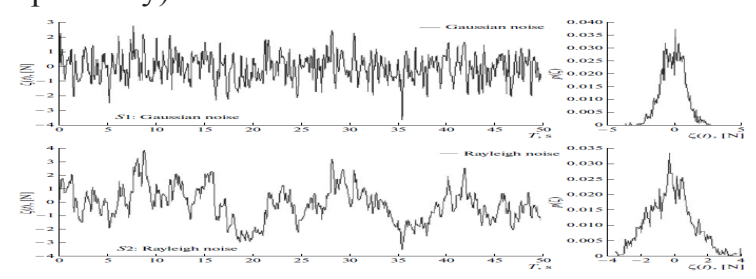

Figure 14. Random noise used in situations (S1, S2)

Physically the first criterion is equivalent to the total energy of the overturned pendulum and the second criterion characterizes the precision of the dynamic behavior of the control object. 
Figure 15 shows $\mathrm{KB} 1$ and $\mathrm{KB} 2$ with the corresponding activated numbers of rules equal to 22 and 33 for a total number of rules of 729 .
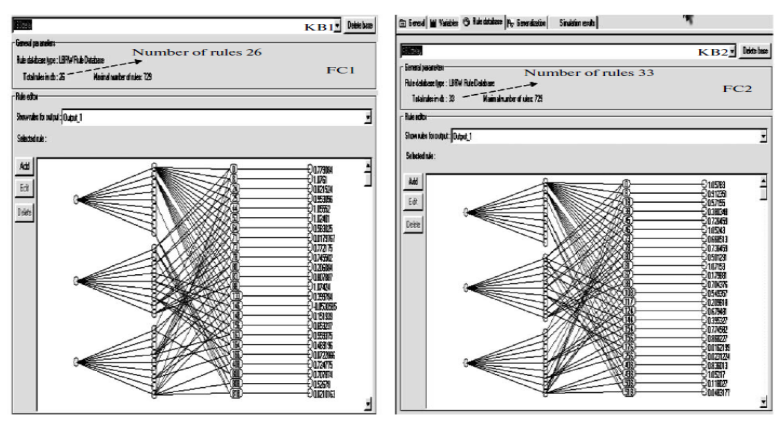

Figure 15. Form of KB1 and KB2 with corresponding activated production rules

Two contingency control situations (S3, S4) were simulated; in one of them (S3) the new noise $\xi(t)$ was introduced, the random signal with uniform one dimensional distribution, the control error signal delay $(0.03 \mathrm{~s})$, and the noise signal in the position sensor of the pendulum (noise amplification coefficient 0.015 ).

Figure 16 shows the example of operation of the quantum FC for formation of the robust control signal using the proportional gain in contingency control situation S3.

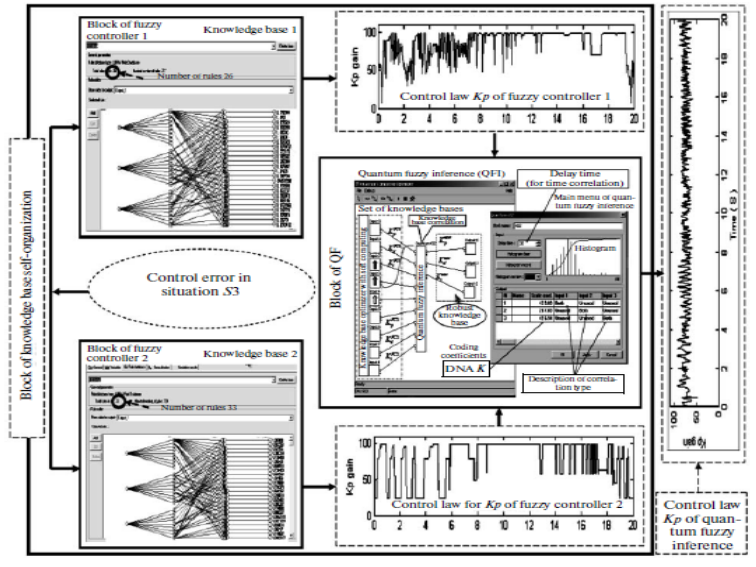

Figure 16. Example of operation of the block of KB self-organization based on QFI

In this case, the output signals of KB1 and KB2 in the form of the response on the new control error in situation $\mathrm{S} 3$ are received in the quantum FC. The output of the block of quantum FC is the new signal for on line control of the factor $k_{p}$.

Thus, the blocks of KB1 and KB2, and quantum FC in Figure 2 form the block of KB self-organization in the contingency control situation.

Figure 17 shows the dynamic behavior of the studied system "cart - pole" and the control laws of the self-organized quantum controller (QFI), FC1 and FC2.

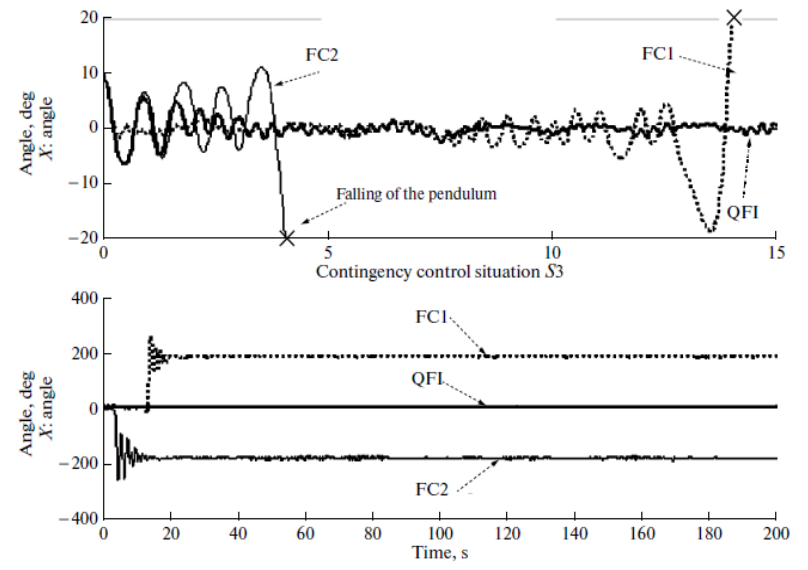

Figure 17. Dynamic motion of pole in situation $S 3$

Remark. The following notation is used in Figure 12 and below: $x=\theta$ is the angle of pendulum deviation from the given position; $z$ is the cart position; the quantum $\mathrm{FC}$ is based on the spatial correlation.

The results of simulation (Figure 17) demonstrate that the dynamic $\mathrm{CO}$ in contingency / unpredicted control situations (S3) for the control of FC1 (FC2) loses stability, and for the control of quantum FC the control system possesses the property of robustness and achieving the control goal is guaranteed. According to the results of simulation (Figure 17), the required amount of control for the given criteria in contingency control situations (S3) for the control of $\mathrm{FC} 1$ and $\mathrm{FC} 2$ also is not achieved, while in the case of control of the quantum FC the control system possesses the required amount of control.

This yields that two non - robust fuzzy controllers can be used to design in on line the robust fuzzy controller using quantum self-organization; the $\mathrm{KB}$ of this robust $\mathrm{FC}$ satisfies both quality criteria.

Therefore, the decomposition of the solution to the above multi-objective optimization problem for the robust $\mathrm{KB}$ in the contingency control situation into partial solutions to optimization sub-problems physically can be performed in on line in the form of separate responses of the corresponding individual KBs optimized with different fixed cost functions and control situations. The aggregation of the obtained partial solutions in the form of the new robust KB is performed based on the quantum FC containing the mechanism of formation of the quantum correlation between the obtained partial solutions. As a result, only responses of the finite number of individual KBs containing limiting admissible control laws in the given contingency situations are used.

The control laws of variation of the gains of the fuzzy PID controller formed by the new robust KB have a simpler physical realization (see, Figure 16, right side col- 
umn), and as a result they possess better characteristics of individual control cost function for the contingency control situation.

For experimental testing a physical model of robot (Figure 18) is used.

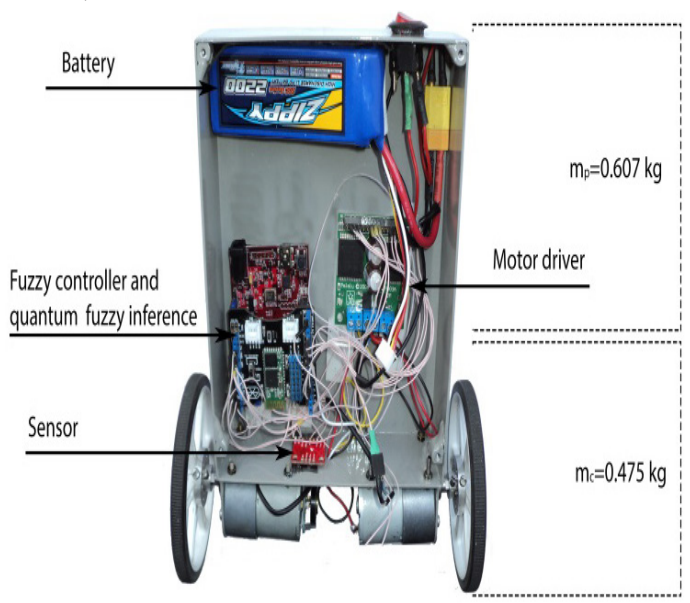

Figure 18. Mobile robot configuration

Three situations of control are tested.

o First situation images simple situation.

o The second situation use uniform noise in control channel, Gaussian noise in wheel friction and delay of control action $-0.01 \mathrm{sec}$.

o And the third situation has delay of control action equal $0.03 \mathrm{sec}$.

Simulation and experimental results (for the complex situation 3) are shown in Figure 19.

PID controller as FC1 and FC2 do not reach the goal in unpredicted situation. But quantum FC based on these fuzzy controllers, successful in unpredicted situation. For experiments and modeling we use QFI with temporal correlation, between $\mathrm{FC} 1$ and $\mathrm{FC} 2$.

Thus, the output signal of the quantum $\mathrm{FC}$ represents the on line optimal control signal for variation of the gains of the fuzzy PID controller which includes the necessary (best) qualitative characteristics of output control signals of each of the fuzzy controllers with priority and dominating component among the control quality criteria.

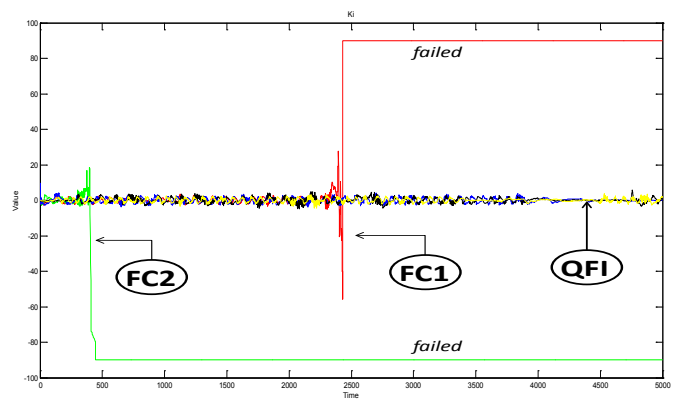

(a)

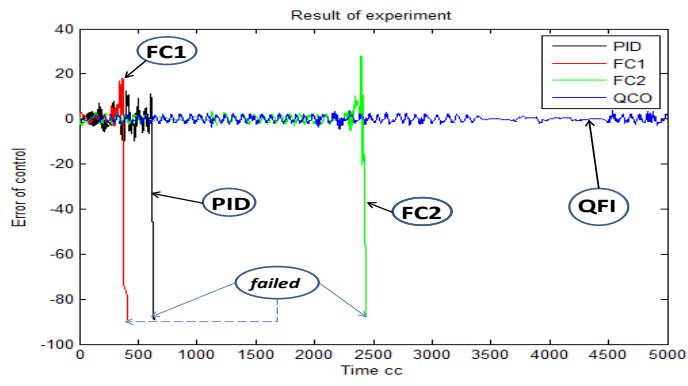

(b)

Figure 19. Control error. Unpredicted situation: (a) modeling; (b) experiment on physical model

PID controller as $\mathrm{FC} 1$ and $\mathrm{FC} 2$ do not reach the goal in unpredicted situation.

But quantum FC based on these fuzzy controllers, successful in unpredicted situation. For experiments and modeling we use QFI with temporal correlation, between $\mathrm{FC} 1$ and $\mathrm{FC} 2$.

Thus, the output signal of the quantum FC represents the on line optimal control signal for variation of the gains of the fuzzy PID controller which includes the necessary (best) qualitative characteristics of output control signals of each of the fuzzy controllers with priority and dominating component among the control quality criteria.

Therefore, the generalized self-organization principle ${ }^{[1-7]}$ is realized.

\subsubsection{Benchmark 2: Remote Rule Base Optimiza- tion}

To compare method of remote rule optimization on the real control object with method using Matlab simulation for optimization we created $6 \mathrm{~KB}-\mathrm{FC}$.

\begin{tabular}{|c|c|c|c|}
\hline No & TS Source & $\begin{array}{c}\text { Optimization } \\
\text { method }\end{array}$ & $\begin{array}{c}\text { Rule's } \\
\text { count }\end{array}$ \\
\hline FC1 & Math. model & Math. modelling & 125 \\
\hline FC2 & CO (GA-PID) & Math. modelling & 125 \\
\hline FC3 & Math. model & Remote connection & 125 \\
\hline FC4 & CO (GA-PID) & Remote connection & 125 \\
\hline FC5 & Math. model & $\begin{array}{c}\text { Math. modeling + Remote } \\
\text { connection }\end{array}$ & 125 \\
\hline FC6 & CO (GA-PID) & $\begin{array}{c}\text { Remote connection + Math. } \\
\text { modeling }\end{array}$ & 125 \\
\hline
\end{tabular}

Experiment and modeling were performed in two control situations. The first situation (S1) is typical for the control system (the initial angle equals to 1). The goal is to maintain the pendulum in equilibrium $\left(0^{\circ}\right.$ angle of deflection). It should be noted that $\mathrm{KB}$ optimization held in this control situation. The second situation is unexpected (S2). The initial angle equals to $5^{\circ}$. This situation characterizes the perturbation caused by external influences on $\mathrm{CO}$.

Figure 20 shows a comparison of integrals of squared 
error for all regarded regulators in a typical situation of control.

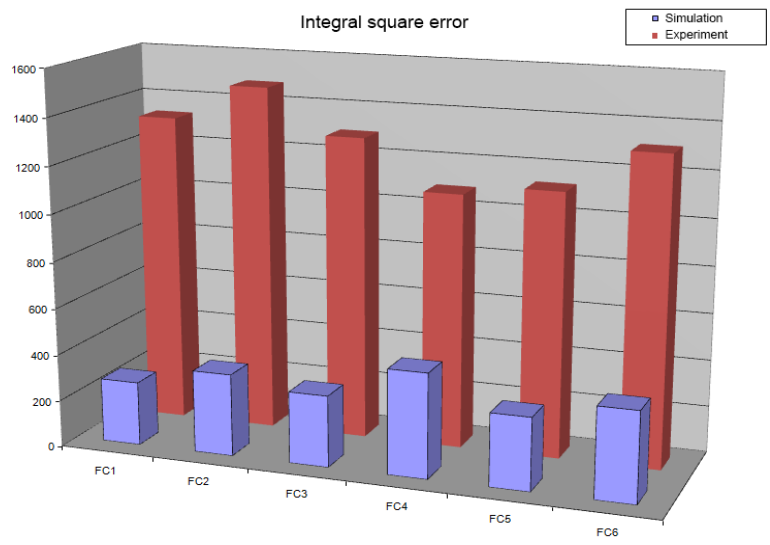

Figure 20. Integral square error. Typical situation: Simulation and experiment.

The lower is integral square error level, the better contr-oller works. Consider the results of simulation and exper-iment in unpredicted situation of control:

Figure 21 shows a comparison of integrals of squared error for all regarded regulators in an unpredicted situation of control.

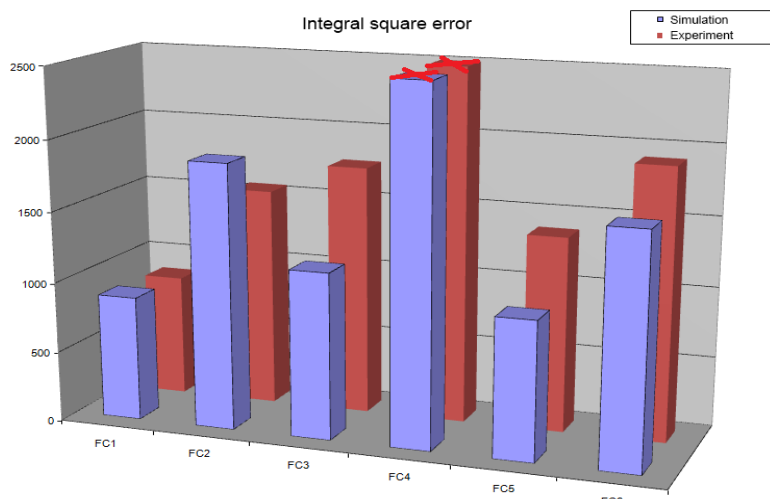

Figure 21. Integral square error. Unpredicted situation: Simulation and experiment.

\subsubsection{Benchmark 3: Remote Quantum Base Optimization}

Let's compare the PID controller, fuzzy controllers FC1 and FC4, and QFI controllers based on different correlations: Quantum-Space (Q-S), Quantum-Time (Q-T), Quantum-Space-Time (Q-ST). These QFI controllers are optimized using remote connection.

Mathematical modeling and physical experiments took place in two control situations:

- in the first (typical) situation (S1), the delay of control is standard as $0.015 \mathrm{sec}$;

- in the second unpredicted situation (S2), the delay of the control as $0.035 \mathrm{sec}$.

From Figure 22 and 23 it can be seen that KB optimi-zation using a remote connection with quantum optimizer can improve the quality of control in a typical and unpredicted situation better than the application only the soft computing technology.
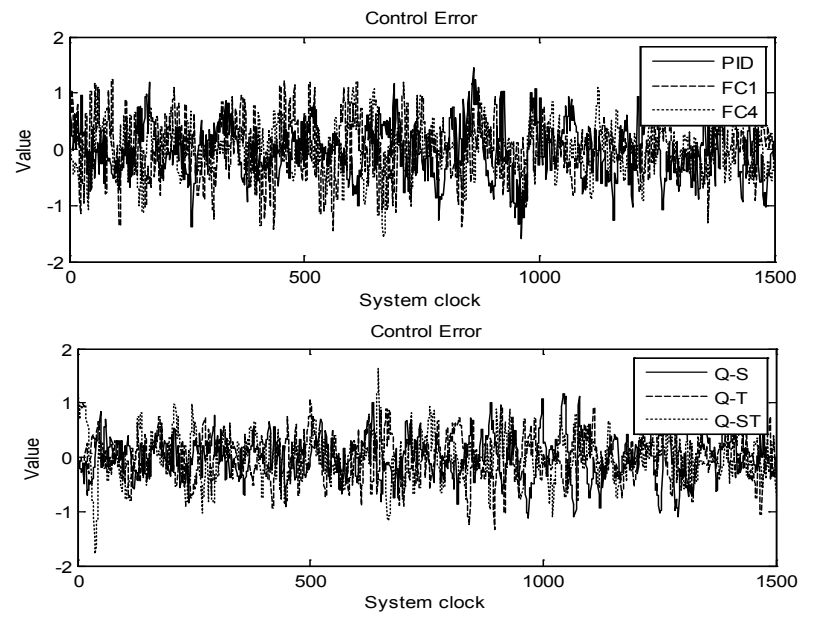

Figure 22. Control error. Typical situation of control (Experiment)
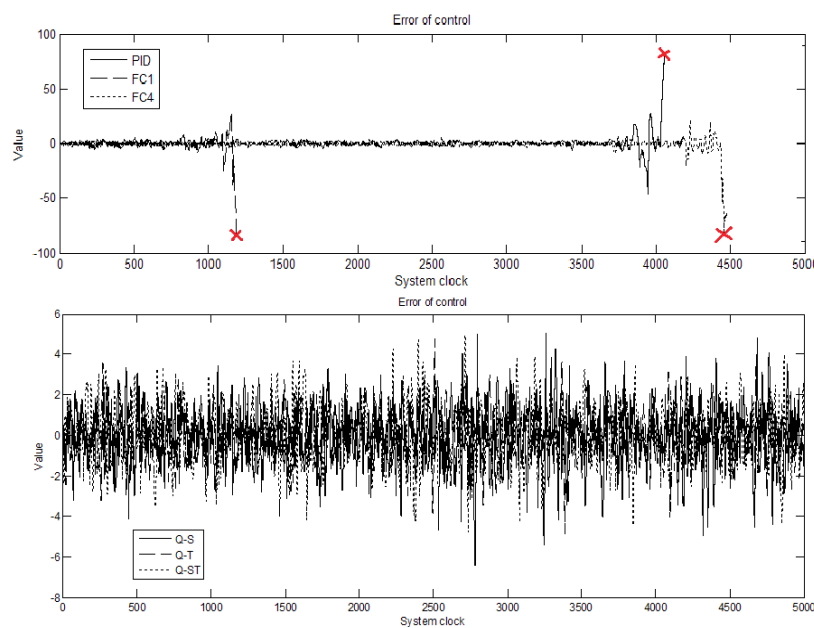

Figure 23. Control error. Unpredicted situation of control (Experiment)

Related works. Quantum computing approaching in robot path planning, emotion design, navigation, learning, decision making was applied also in ${ }^{[6,7,35,36]}$ etc. Our approach is based on quantum self-organization of knowledge bases using responses of imperfect KB from fuzzy controllers on unpredicted situations in on line.

\subsection{Benchmark's Simulation of Wise Control with QFI Based on QGA}

One of the interesting ideas was proposed in 2004, taking the first steps in implementing the genetic algorithm 
on a quantum computer ${ }^{[3,36]}$. The author proposed this quantum evolutionary algorithm, which can be called the reduced quantum genetic algorithm (RQGA).

The algorithm consists of the following steps: 1) Initialization of the superposition of all possible chromosomes; 2) Evaluation of the fitness function by the operator $F ; 3$ ) Using Grover's algorithm; 4) Quantum oracle; 5) Using of the diffusion operator Grover G; 6) Make an evaluation of the decision. The search for solutions in RQGA is performed in one operation.

In this case the matrix form is the result of RQGA action as following (see Figure 24)

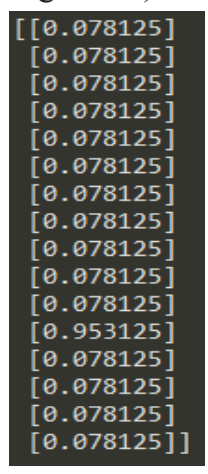

Figure 24. The result of the RQGA algorithm

After action of GQA more than 1000 generation we can see in Figure 25 that around 70\% spatio-temporal correl-ation have best probability choice.

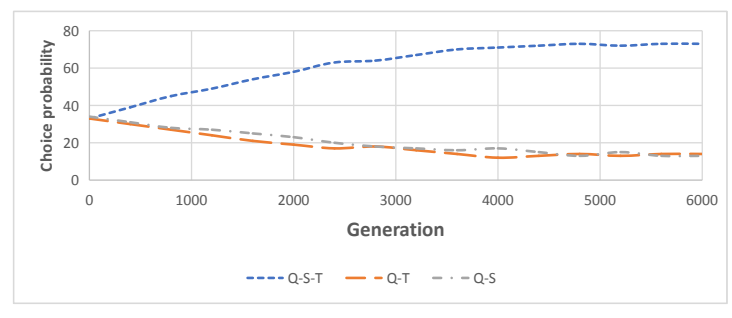

Figure 25. The result of the QGA

Temporal and spatial correlations have similar quality. After 5000 generations probability value is not changing. QGA after 200 generations the probability choice of spatio-temporal correlation decreases to $60 \%$ (see, Figure 26).

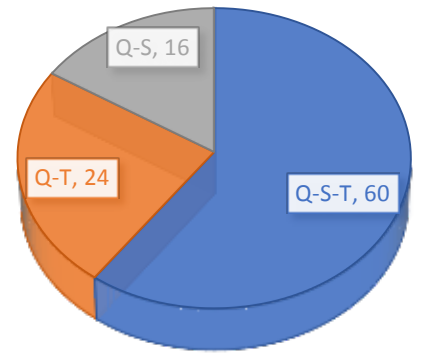

Figure 26. The result of the quantum genetic algorithm 200 times
The overall strategy for improving the quality of QGA is to use small improvements in the algorithm. For example, including new operators: "quantum disaster", disturbance, or other customized algorithms ${ }^{[7]}$. But in many cases, these operators are only useful in highly specific applications.

\subsubsection{Simulator Structure and Examples of Appli- cations}

The use of simulators has long been used in various industries: motor racing, aviation, surgery and many others. The development of virtual reality technology and augmented reality adds the ability to create simulators with full immersion. Most of the server side work is math. It is necessary to calculate the position of the carriage, the angle of inclination of the pendulum in space. For this reason, Python and the Django framework, which implements the model-view-controller (MVC) approach, were chosen as the programming language (or in Django, this is the model-view-template (MVT)). MySQL is used to store all data, and the architecture has been developed for adding Redis to be faster, if the MySQL operation speed is insufficient.

Figure 27 shows Benchmark results of quantum intell-igent control simulation of "cart - pole" system with QGA (box for the type choice of "Quantum correlation" on Figure 4).

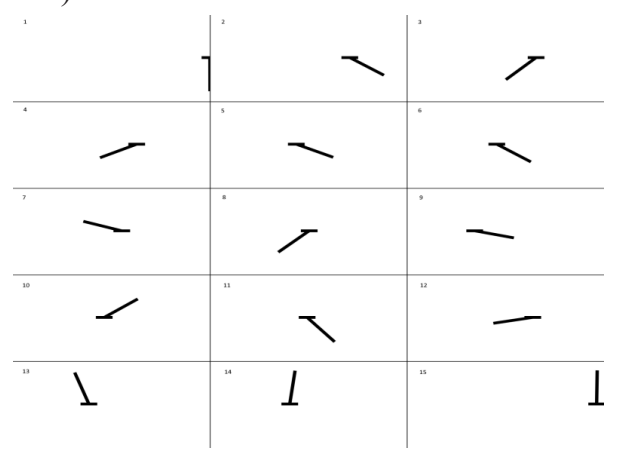

(a) visualization of inverted pendulum behavior

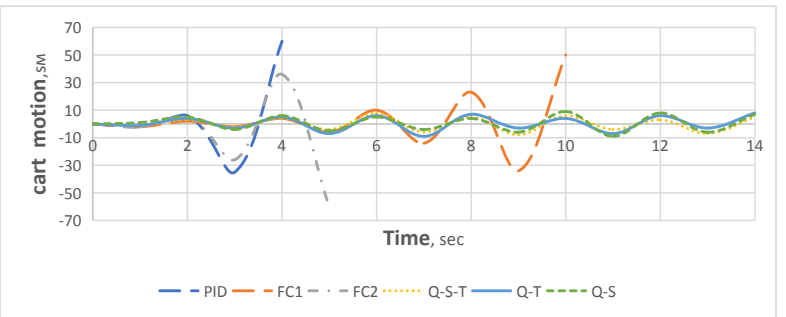

(b) cart behavior (Q-S-T - quantum spatio-temporal correlation; Q-T - quantum temporal correlation; Q-S quantum spatial correlation; FC - fuzzy controller)

Figure 27. Simulation results of "cart - pole" system with PID - controller, fuzzy controllers and QFI-QGA-controller with different quantum correlation 
Partial rendering performance of the simulator is shown in Figure 27. The described method is differed from others results described in ${ }^{[7,8]}$.

Example: Application of quantum computing optimizer of knowledge base (QCOPTKB ${ }^{\mathrm{TM}}$ ) for the case of experimental teaching signal from control object. Control object shown in Figure 28a.

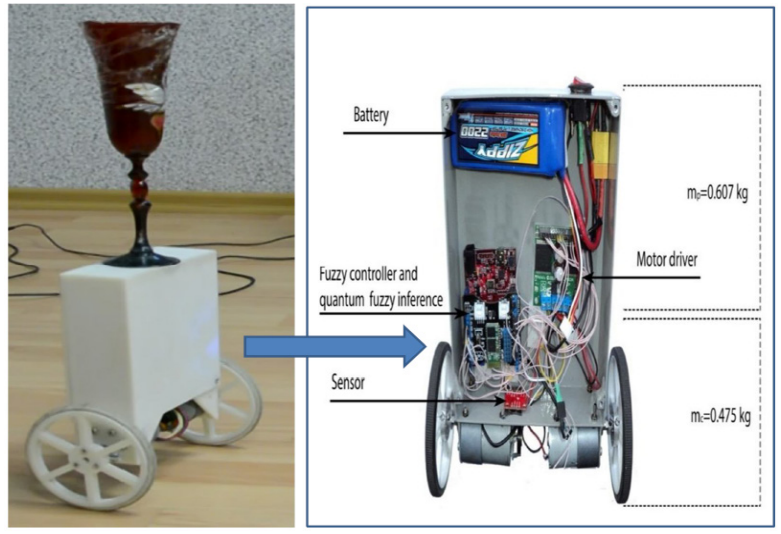

(a)

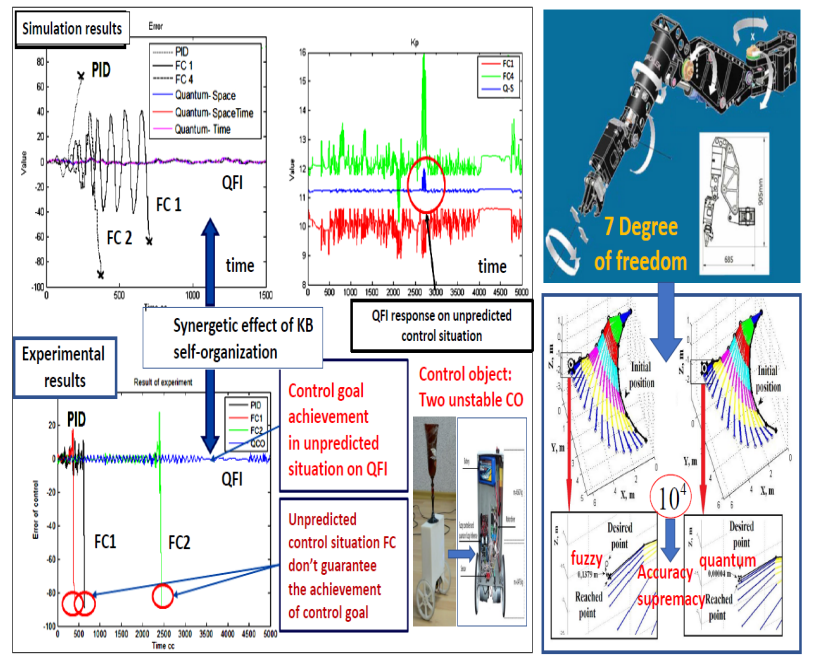

(b)

Figure 28. Autonomous robot with inverted pendulum (a) and simulation \& experimental results comparison for unpredicted control situation in cases of PID-controller, fuzzy controller and QFI-controller for "cart - pole" system and 7 Dof redundant robotic manipulator (b)

Structure of robust intelligent control system (ICS) based on QFI is shown in Figure 2 and on Figure 4 is shown QAG structure of QFI that used in the simulation and experiment. Figure $28 \mathrm{~b}$ demonstrated results of simulation and experimental results comparison. Mathematical modeling and experimental results are received for the case of unpredicted control situation and knowledge base of fuzzy controller was designing with SW of QCOPTKB ${ }^{\mathrm{TM}}$ for teaching signal measured directly from control object (autonomous robot in Figure 28a, Box $Z^{-1}$ on Figure 2). As model of unpre- dicted control situation on Figure 2 (Box $Z^{-1}$ ) was the situation of feedback sensor signal delay on three times.

For robotic manipulator with 7 DoF result demonstrated on Figure 28b (right side) and the accuracy of quantum controller is more than ten thousand times higher of soft computing approach. It is the demonstration of quantum soft computing supremacy.

Results of controllers behavior comparison confirm the existence of synergetic self-organization effect in the design process of robust $\mathrm{KB}$ on the base of imperfect (non robust) $\mathrm{KB}$ of fuzzy controllers on Figure 28. In unpredicted control situation control error is dramatically changing and $\mathrm{KB}$ responses of fuzzy controllers (FC 1 and FC 2) that designed in learning situations with soft computing are imperfect and do not can achieve the control goal. Using responses of imperfect KB (as control signals for designing the schedule of time dependent coefficient gain in PID-controller on Figure 2) in Box QFI the robust control is formed in on line. This effect is based on the existence of additional information resource that extracted by QFI as quantum information hidden in classical states of control signal as response output of imperfect KB's on new control error (QFI algorithm structure on line in Figure 4). QGA in Figure 5 for this case recommended the spatial quantum correlation as was early received in $[1,2]$.

\section{Discussion}

The described design method of ICS based on QAG-approach let to achieve global robustness in the case of unpredicted control situations in online using new types of computational intelligence toolkit as quantum and soft computing and based on computational resource of classical computers. The introduced model of QFI is a new type of quantum search algorithm based on sophisticated structure of quantum genetic algorithm embedded in its structure. Such on an approach to the solution of robust control design problems of classical nonlinear control objects (in general globally unstable and essentially nonlinear) is considered as benchmark for effective application of developed design information technology of ICS ${ }^{[4,6-8]}$. The results of simulation and experiment show unconventional (for classical Boolean logic) conclusion: from response of two non-robust imperfect KB of FCs in the structure of ICS on Figure 2 with new quantum search algorithm QFI based on QGA possible to design in online robust quantum $\mathrm{FC}$.

With RQGA based on reduced Grover's QSA used spatial quantum correlation between two coefficient gain schedules of FCs in Figure 2 and quantum self-organization of imperfect KBs in online effectively on classical standard chip realized and described on concrete example. 
This synergetic information effect has pure quantum nature, used hidden in classical states quantum information as additional information resource and does not have classical analogy.

Remark. Figure 29 demonstrates one of new possible approaches to design intelligent cognitive control system applying quantum robotic communications and BCI-system as in case of a chess game with quantum - like decision making.

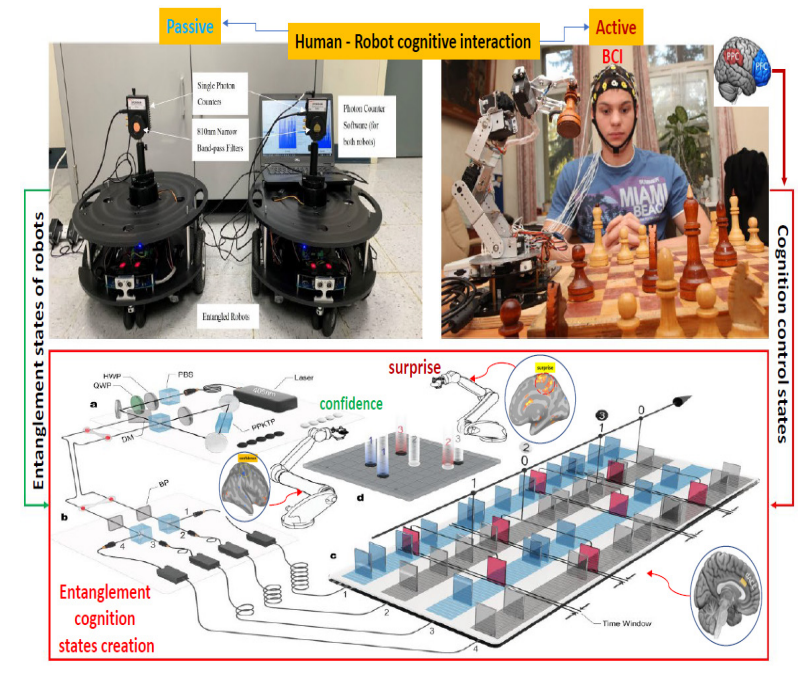

Figure 29. Prototype of hybrid intelligent cognitive control system with $\mathrm{BCI}$ and robotic quantum-like decision making systems

Future applications are correlated with the development of hybrid intelligent cognitive emotion control system as the cognitive control system based on "Brain - Computer - Interface" (BCI) and quantum - like decision making models ${ }^{[57]}$ applying robotic - human interactions with quantum knowledge exchanging and communications as "brain-to-brain" and so on. These approaches apply quantum deep machine learning with quantum neural network and quantum genetic algorithms for designing the synergetic information control of intelligent robotic swarm with entangled states and knowledge exchanging, and free energy principle of human being emotion with minimum generalized entropy production ${ }^{[58]}$. In Part II of this article the applications of intelligent cognitive control system presented on Figure 29 discussed.

\section{Conclusions}

o Self-organized man-made intelligent port-controlled Hamiltonian systems are described as quantum control algorithm.

o Structure of QFI model ${ }^{[35]}$ based on QGA as the particular case of general quantum control algorithm of self-organization is introduced. o Quantum supremacy of intelligent cognitive control with information-thermodynamic trade-off distribution ${ }^{[36]}$ of main control qualities for micro-nanorobotics demonstrated.

o New circuit implementation design method of quantum gates for fast classical efficient simulation of QAs is developed. Benchmarks of design application as Grover's QSA and QFI based on QGA demonstrated. Applications of QAG approach in intelligent control systems with quantum self-organization of imperfect knowledge bases are described on concrete examples.

o The results demonstrate the effective application possibility of end-to-end quantum technologies and quantum computational intelligence toolkit based on quantum soft computing for the solution of intractable classical and algorithmically unsolved problems as design of global robustness of ICS in unpredicted control situations and intelligent robotics.

o Efficient simulation on classical computer quantum soft computing algorithms, robust fuzzy control based on quantum genetic (evolutionary) algorithms and quantum fuzzy neural networks (that can realize as modified Grover's QSA), AI-problems as quantum gate simulation approaches and quantum deep learning, quantum optimization in Part II are considered.

o Thus, positive application results of mutual technologies based on soft and quantum computing give the possibility of application Feymann - Manin thesis to study classical physical system as inverse problem "quantum control system - classical control object" solve effectively classical intractable and algorithmic unsolved problems.

\section{References}

[1] Litvintseva, L. V., Ulyanov, I. S., Ulyanov, S. V., Ulyanov, S. S. Quantum Fuzzy Inference for Knowledge Base Design in Robust Intelligent Controllers [J]. Journal of Computer and Systems Sciences Inter-national, 2007, 46(6): 908-961.

DOI: $10.1134 / \mathrm{S} 1064230707060081$.

[2] Litvintseva, L. V., Ulyanov, S. V. Intelligent Control Systems. I. Quantum Computing and Self-Organization Algorithm [J]. Journal of Computer and Systems Sciences International, 2009. 48. (6): 946-984. DOI: $10.1134 / \mathrm{S} 1064230709060112$.

[3] Ulyanov, S. V. Intelligent Robust Control System Based on Quantum KB-Self-organization: Quantum Soft Computing and Kansei / Affective Engineering Technologies [J]. Springer International Publishing, 2014. pp. 37-48.

[4] Ulyanov, S.V. Intelligent self-organized robust control design based on quantum/soft computing 
technologies and Kansei Engineering [J]. Computer Science J. of Moldova, 2013, 21 (2(62)): 242 - 279.

[5] Ulyanov, S.V. Self-organizing quantum robust control methods and systems for situations with uncertainty and risk [P]. Patent US 8788450 B2, 2014.

[6] Ulyanov, S. V. Self-organized robust intelligent control [M] Saarbrücken: LAP Lambert Academic Publishing, 2015. $412 \mathrm{p}$.

[7] Ulyanov, S.V. Quantum relativistic informatics [M]. LAP LAMBERT Academic Publishing, OmniScriptum GmbH \& Co. KG, 2015.

[8] Ahmadi, B., Salimi, S., Khorashad, A.S., Kheirandish, $\mathrm{F}$. The quantum thermodynamic force responsible for quantum state transformation and the flow and backflow of information [J]. SCIENTIFIC REPORTS, 2019, 9 (8746) https://doi.org/10.1038/ s41598-019-45176-1.

[9] Ahmadi, B., Salimi, S., Khorashad, A.S. Irreversible work and Maxwell demon in terms of quantum thermodynamic force [J]. SCIENTIFIC REPORTS, 2021, 11 (2301) https://doi.org/10.1038/s41598-02181737-z.

[10] Zhang, K., Wang, X., Zeng, Q. et al. Conditional Entropy Production and Quantum Fluctuation Theorem of Dissipative Information [R]. arXiv:2105.06419v1 [quant-ph] 13 May 2021.

[11] Nakamura, T., Hasegawa, H.H., Driebe, D.J., Reconsideration of the generalized second law based on information geometry [J]. J. Physics Communications, 2019, 3: $015015 \mathrm{https} / / /$ doi.org/10.1088/2399-6528/aafe1b.

[12] Sieniutycz, S., et all. Framework for optimal control in multistage energy systems [J]. Physics Reports, 2000, 326(2).

[13] Ulyanov, S.V. System and method for control using quantum soft computing [P]. Patent, US 6,578,018, B1, 2003.

[14] van der Schaft, A.J. Theory of port-Hamiltonian systems (Lectures 1, 2, and 3) [M]. Network Modeling and Control of Physical Systems. DISC, 2005.

[15] van der Schaft, A., Jeltsema, D. Port-Hamiltonian Systems Theory: An Introductory Overview. Founda-tions and Trends R in Systems and Control [M]. 2014. 1(2-3): 173-378. doi.org/10.1561/2600000002.

[16] Moxley, III. Quantum Port-Hamiltonian Network Theory: Universal Quantum Simulation with RLC Circuits [R]. Dartmouth College, Dartmouth Digital Commons. Hanover, NH 03755 USA. 2020.

[17] Marcolli, M. Motivic information [R]. arXiv:-1712.08703v1 [math-ph] 23 Dec 2017.

[18] Combe, N.C., Manin, Yu.I., Marcolli, M. Geometry of information: classical and quantum aspects [R]. 2021 [available http: www.its.caltech.edu].
[19] Tribus, M., Shannon, P.T., Evans, R.B. Why thermo-dynamics is a logical consequence of information theory [J]. A. I. Ch. E. Journal. 1966, 12( 2): 244 248.

[20] Bais, F.A., Farmer, J.D. Physics of information [R]. SFI WORKING PAPER: 2007-08-029. [www. santafe.edu]. The Handbook on the philosophy of information, Eds by J. van Benthem and P. Adriaans. 2009.

[21] Sagawa, T., Ueda, M. Minimal Energy Cost for Ther-modynamic Information Processing: Measurement and Information Erasure[J]. Phys. Rev. Lett., 2009, 102(25): 250602. [Erratum. Phys. Rev. Lett. 106, 189901, 2011.].

[22] Horowitz, J. M., Sandberg, H. Second-law-like inequalities with information and their interpretations [J]. New Journal of Physics, 2014, 16: 125007.

[23] Sandberg, H, et al. Maximum work extraction and implementation costs for nonequilibrium Maxwell's demon [J]. Physical Review E, 2014, (4): pp. 042119.

[24] Haddad, W. M., Chellaboina, V., Nersesov, S. G. Thermodynamics: A Dynamical Systems Approach [M]. Princeton Series in Applied Mathematics Princeton. NJ: Princeton University Press, 2005.

[25] Fukuda, T., Kawamoto, A., Arai, F. Micro mobile robot in fluid, $2^{\text {nd }}$ report, Acquisition of swimming motion by RBF Fuzzy neuro with unsupervised learning [J]. Trans. Japan Society of Mechanical Engineers, 1995, 61(591): 274-279.

[26] Ulyanov, S.V., Fukuda, T. et al. Quantum and thermodynamic conditions for artificial life of biological mobile micro-nano-robot with AI control (Report 2) $[C]$. Proc. $7^{\text {th }}$ Int. Symposium on Micromachine and Human science, 1996, Nagoya, Japan: 241-248.

[27] Ulyanov, S.V., Rizzotto, G.G., Fukuda, T. et al. Advanced intelligent control systems in non-linear mechatronics and robotics: From macro-to-micro-systems [C]. Proc. of Eur. Conference on Circuit Theory and Design (ECCTD'99), 1999, Stresa, Italy, 2: pp. 983-986.

[28] Xiao, J., Yang, L. Thermodynamic properties of $\alpha$ -helix protein: a soliton approach [J]. Phys. Rev., 1991, 44A(12): 8375-8379.

[29] Bolterauer, H., Tuszynski, J.A., Sataric, M.V. Frölich and Davydov regimes in the dynamic of dipolar oscillations of biological membranes [J]. Phys. Rev., 1991, 44A(2): 1366-1381.

[30] Ravazy, M. Equation of motion approach to the problem of damped motion in quantum mechanics [J]. Phys. Rev., 1990, 44A(3): 1211-1217.

[31] Schuch, D. Nonunitary connection between explicitly time-depended and nonlinear approaches for the 
description of dissipative quantum systems [J]. Phys. Rev., 1992, 55A(2): 935-940.

[32] Ulyanov, S.V. Dynamic systems with fuzzy and random time-variant structures (stochastic vibrations, coherent states and solitons in classical, relativistic and quantum control systems) [M]. Eng. Cybernetics. 1992. Vol. 15. pp. 3-145.

[33] Korsh, H.J., Steffen, H. Dissipative quantum dynamics, entropy production and irreversible evolution towards equilibrium [J]. J. Phys., 1987, 20A(12): 37873803.

[34] Korsh, H.J., Steffen, H. Dissipative quantum dynamics: solution of the generalized von Neumann equation for the damped harmonic oscillator $[\mathrm{J}]$. J. Phys., 1992, 25(7): 2043-2064.

[35] Ulyanov, S.V., Litvintseva, L.V. Design of self-organized intelligent control systems based on quantum fuzzy inference: Intelligent system of systems engineering approach [C]. IEEE International Conference on Systems, Man and Cybernetics. 2005. Hawaii, USA, 10-12 Oct. 2005, 4: 3835-3840.

[36] Ulyanov, S. V. Quantum fast algorithm computational intelligence PT I: SW / HW smart toolkit [J]. Artificial Intelligence Advances, 2019, 1(1): 18-43.

[37] Ito, S. Thermodynamics of information geometry as a generalization of the Glansdor-Prigogine criterion for stability [R]. arXiv:1908.09446v1 [cond-mat.statmech] 26 Aug 2019.

[38] Brandão, F. The second laws of quantum thermodynamics [J]. PNAS, 2015, 112(11): 3275-3279. doi/10.1073/pnas.1411728112.

[39] Zozor, S. On Generalized Stam Inequalities and Fisher-Rényi Complexity Measures [J]. Entropy, 2017, 19(493): 1-31.

DOI: $10.3390 / \mathrm{e} 19090493$.

[40] Robinett, R. D., Wilson, D. G. Exergy and Irreversible Entropy Production Thermodynamic Concepts for Control Design: Nonlinear Systems [C]. $14^{\text {th }}$ Mediterranean Conf. on Control and Automation, 2006: $1-8$.

DOI: 10.1109/MED.2006.328728.

[41] Sagawa, T., Masahito, U. Generalized Jarzynski Equality under Nonequilibrium Feedback Control [J]. Phys. Rev. Lett., 2010, 104: 090602.

DOI: 10.1103/PhysRevLett.104.090602.

[42] Goold, J. The role of quantum information in thermodynamics - a topical review [J]. J. Phys. A: Math. Theor., 2019, 49: 143001 (50pp).

DOI: $10.1088 / 1751-8113 / 49 / 14 / 143001$.

[43] Vanchurin, V. The World as a Neural Network [J]. Entropy, 2020, 22(1210).

DOI: $10.3390 / \mathrm{e} 22111210$.
[44] Sagawa, T. Thermodynamic and logical reversibilities revisited [R]. arXiv: 131П2.1886v1 [cond- mat. stat-mech] 8 Nov 2013.

[45] Yamano, T. Phase space gradient of dissipated work and information: A role of relative Fisher information [R]. arXiv: 131П2.2176v1 [cond-mat.stat-mech] 9 Nov 2013.

[46] Ilgin, I., Yang, I-Sh. Energy carries information [R]. arXiv:1402.0878v1 [hep-th] 4 Feb 2014.

[47] Horowitz, J. M., Esposito M. Thermodynamics with continuous information flow [R]. arXiv:1402.3276v2 [cond-mat.stat-mech] 14 Feb 2014.

[48] Renes, J. M. Work Cost of thermal operations in quantum and nano thermodynamics [R]. arXiv:1402.3496v1 [math-ph] 14 Feb 2014.

[49] Horowitz, J. M. Sagawa, T. Equivalent definitions of the quantum nonadiabatic entropy production [R]. arXiv:1403.7778v1 [quant-ph] 30 Mar 2014.

[50] Lang, A.H., Fisher, Ch.K., Mehta, P. Thermodynamics of statistical inference by cells [R]. arXiv:1405.4001v1 [physics.bio-ph] 15 May 2014.

[51] Apollaro, T. J. G., Francica, G., Paternostro M., Campisi, M. Work statistics, irreversible heat and correlations build-up in joining two spin chains [R]. arXiv: 1406.0648v1 [cond-mat.stat-mech] 3 Jun 2014.

[52] Gomez, C. Complexity and time [J]. Phys. Rev., 2020, D 101: 065016.

[53] Funo, K., Watanabe, Yu., Ueda, M. Thermodynamic work gain from entanglement [J]. Phys. Rev., 2013, A88(5): 052319.

[54] Toyabe, S., Sagawa, T., Ueda, M., Muneyuki, E., Sano, M. Experimental demonstration of information-to-energy conversion and validation of the generalized Jarzynski equality [J]. Nature Physics, 2010. 6: 988-992.

[55] Van der Meer, R., Ng, N. H. Y., Wehner, S. Smoothed generalized free energies for thermodynamics $[\mathrm{J}]$. Phys. Rev. A, 2017, 96(6): Pp. 062135.

[56] Mirkes, E.M. Universal Gorban's Entropies: Geometric Case Study. [J]. Entropy, 2020, 22(3):264; https:// doi.org/10.3390/e22030264.

[57] Khrennikov, A. Quantum-like model for unconscious-conscious interaction and emotional coloring of perceptions and other conscious experiences [R]. arxiv.org/abs/2106.05191v1 [q-bio. NC] 6 June 2021.

[58] Korenkov, V.V., Ulyanov, S.V., Shevchenko, A.A., Shevchenko, A.V. Intelligent cognitive robotic: Quantum cognitive computing technologies [M]. M.: Kurs, 2021. 


\section{Appendix 1. Interrelation between principles of max- imum work value generation and minimum entropy production: Hamilton-Jacobi equation approach}

Power of information-thermodynamic approaches for the analysis of dynamic systems demonstrated in many publications ${ }^{[8-12,17-24,37-55]}$. This Appendix 1 concentrate the attention on quantum supremacy of the extraction work from quantum hidden information that applied as the information-thermodynamic force in intelligent cognitive controllers embedded in the structure of smart control systems.

The definition principle of system maximal work gen-eration is the following:

$$
W_{\max }=\max \left\{-\int_{T^{i}}^{T^{f}} L(T, \dot{T}) d \tau\right\}=\max \left\{-\int_{T^{i}}^{T^{f}} c\left(1-\frac{T^{e}}{T+\dot{T}}\right) \dot{T} d \tau\right\}
$$

In (A1.1) the function $L(T, \dot{T})$ is a Lagrangian function of a dynamic system.

The alternative definition form of work is the functional as following:

$$
\begin{aligned}
W_{\left[T^{i}, T^{f}\right]} \equiv \frac{W}{G} & =\int_{T^{i}}^{T^{f}}-c\left(1-\frac{T^{e}}{\dot{T}}\right) d \tau-T^{e} \int_{T^{i}}^{T^{f}} c \frac{\dot{T}^{2}}{T(T+\dot{T})} \\
& =-\int_{T^{i}}^{T^{f}} c\left(1-\frac{T^{e}}{\dot{T}}\right) d T-T^{e} S
\end{aligned}
$$

and

$$
S=\int_{T^{i}}^{T^{e}} c \frac{\dot{T}^{2}}{T(T+\dot{T})} d \tau
$$

Thus, problems of the maximum released work is equivalent to the associated problem of the minimum entropy production and have the same solutions. The characteristic function

$$
I\left(\tau^{f}, T^{f}, \tau^{i}, T^{i}\right) \equiv \max W_{\left[T^{i}, T^{f}\right]}=\max \left\{\int_{T^{i}}^{T^{f}}\left[-c\left(1-\frac{T^{e}}{T+u}\right) u\right] d \tau\right\}(
$$

control the maximum work delivery. It is clear that the quantity $I$ in Equation (A1.4) describes the extremal value of the work $W\left(T^{i}, T^{f}\right)$ of Equation (A1.1) as following:

$$
W_{\max }=\max \left\{-\int_{T^{i}}^{T^{f}} L(T, \dot{T}) d \tau\right\}=\max \left\{-\int_{\tau^{i}}^{\tau^{f}} c\left(1-\frac{T^{e}}{T+\dot{T}}\right) \dot{T} d \tau\right\}
$$

whose extremal value is the function $I\left(T^{i}, \tau^{i}, T^{f}, \tau^{f}\right)$.

The Hamilton-Jacobi equation for the maximum work problem has described as

$$
\begin{gathered}
\frac{\partial I}{\partial \tau}+H\left(T, \frac{\partial I}{\partial \tau}\right)=0 \\
H\left(T, \frac{\partial I}{\partial T}\right)=c\left[\sqrt{T^{e}}-\sqrt{T\left(1-\frac{1}{c} \frac{\partial I}{\partial T}\right)}\right]^{2}
\end{gathered}
$$

Therefore, the variational front-end problem for the maximum work $W$ is equivalent to the variational fixedend problem of the minimum entropy production.

Example: Thermodynamic work extraction with information gain. During the isothermal expansion, work is performed by the particle while moving the barrier (which could be extracted by e.g. attaching a pulley and weight to the barrier). The total work performed during the isothermal expansion is given by $W_{\text {exp }}=\int_{V / 2}^{V} \frac{k_{B} T}{V} d V=k_{B} T \log 2$ and this work is gained by a full conversion from heat energy to work. Conservation of energy shows that, since the initial and final state is identical with the same energy, $W$ $=-Q$. If the heat capacity of the environment was not infinite, the work extracted per cycle would be smaller than $k_{\mathrm{B}} T \log 2$ since the final state would have lower energy than the initial one. Nevertheless, by continually repeating the process the heat bath would eventually be completely drained of energy, all of which would be converted to useful work. Szilard emphasized the necessity of performing a measurement for the engine to work. He connected the apparent violation of the second law with the state of the demon's knowledge, and believed that their solution to the paradox was due to some hidden entropic cost associated with the measurement.

There are three main approaches to explain the apparent violation of the second law that Maxwell's demon and the Szilard engine implies.

1. The first approach focuses on the role of fluctuations, which are usually ignored in the idealized thought-experiments but will always be present in real systems.

2. The second approach follows Szilard's own belief and focuses on the entropic cost of performing measurements.

3. The third approach focuses on the fact that the demon has to store the information it obtains about the system. Unless this information is deleted, the final state of the universe will not be identical to the initial state. The third approach focuses on the cost of deleting this memory.

The second law of thermodynamics places constraints on state transformations. A proper definition of the second law of thermodynamics is that entropy cannot spontaneously decrease when averaged over a suitable timescale. What constitutes a suitable time-scale depends on the relaxation time of the system in question and is difficult to define in a general way. A quantitative description of the connection between fluctuations and the second law was given in 1993, when Evans et. al introduced the fluctuation theorem. The theorem is actually a group of closely connected theorems, one of which relates the probability of observing a time-averaged entropy production of magnitude $\Delta S$, to the probability of that it takes the opposite value $-\Delta S$ as following $\frac{P(\Delta S)}{P(-\Delta S)}=\exp \left\{\frac{\Delta S}{k_{B}}\right\} \geq 1$. Since the right side is always 
positive and larger than 1 , the probability to observe fluctuations that temporarily "violate" the second law is always less or equal to the ones that obey it. Moreover, the relative probability of producing and consuming entropy increases exponentially with the amount of entropy change. Since entropy is extensive, the fluctuation theorem also shows that the probability to observe negative entropy fluctuations goes to zero for macroscopic systems. If $Q$ is the average heat absorbed by the system under the transformation, the total entropy production (i.e., system + environment) is then given by

$$
\Delta S_{t o t}=\underbrace{\left(S_{f}-S_{i}\right)}_{\Delta S}-\beta Q \text {. }
$$

According to the second law of thermodynamic, total entropy change is bounded below at zero $\Delta S_{\text {tot }} \geq 0 \rightarrow \Delta S \geq \beta Q$. A physical process which achieves equality in this bound, is considered a thermodynamically reversible process. Notice that the flow of entropy between system and bath is possible for reversible processes, if the amount of heat absorbed by the system is equal to its entropy change. This is because the absorption of heat by the system results in a decrease in the environment entropy according to $\Delta S_{e n v}=-\beta Q$.

Example: Quantum thermodynamic force and work extraction. It is known that negative work $\Delta W$ can be extracted from an isothermal cycle with a feedback controller and due the measurement the change in the entropy of the system can be expressed as $\Delta S_{\text {meas }}=H(X \mid M)-H(X)=-I(X: M)$ and $H(X)=-\sum_{x} \rho(x)$ $\ln \rho(x)$ is the Shannon entropy and $I(X: M)$ Is the mutual information between the state of the system and the measurement $M$ outcome. Thus, while the amount of mutual information $I(X: M)$ is positive then the controller causes the entropy of the system to decrease. It is means that the presence of the controller expected to lead to extracting more work from the system that what expected. The controller can be incorporated into the Second Law as the following

$$
\Delta W \geq \Delta F^{\beta}-\frac{1}{\beta} I\left(\rho_{1}: X\right) \text { and } I\left(\rho_{1}: X\right)=\frac{1}{\beta}\left[S\left(\rho_{1}\right)-H\left(\left(p_{k}\right)\right)\right.
$$

$\left.+H\left(\rho_{1}: X\right)\right]$,

where $\rho_{1}$ is the state of the system at some time $t_{1}$, $S\left(\rho_{1}\right)$ is the von Neumann entropy, $H\left(p_{k}\right)=-\sum_{k} p_{k} \ln p_{k}$ is the Shannon entropy content and

$$
H\left(\rho_{1}: X\right)=-\sum_{k} \operatorname{Tr}\left[\sqrt{D_{k}} \rho_{1} \sqrt{D_{k}} \ln \sqrt{D_{k}} \rho_{1} \sqrt{D_{k}}\right] \quad,
$$$$
\left(D_{k}\right)=M_{k}^{\dagger} M_{k}, p_{k}=\operatorname{Tr}\left[D_{k} \rho\right]
$$

are positive operator value-measure. The time derivative of these three terms give the result for extraction of work with controller as following:

$\frac{d W_{i d r}^{\text {contr }}}{d t}=\frac{1}{\beta}\left[\operatorname{Tr}\left(\dot{\rho}_{1} \ln \rho_{1}\right)\right]+\sum_{k} \dot{p}_{k} \ln p_{k}-\sum_{k} \operatorname{Tr}\left[\sqrt{D_{k}} \dot{\rho}_{1} \sqrt{D_{k}} \ln \sqrt{D_{k}} \rho_{1} \sqrt{D_{k}}\right]$.

Thus, there are three quantum thermodynamic forces responsible for the extra work done during the process. Since the work is given by the change in the internal, we can obtain

$$
W=\Delta F(\alpha)+\alpha^{-1} D_{K L}\left[\rho_{T} \| \rho_{c a n, T}(\alpha)\right]-\alpha^{-1} D_{K L}\left[\rho_{0} \| \rho_{c a n, 0}(\alpha)\right]
$$

where an inverse temperature as $\alpha$, the conservation of the Gibbs-Shannon entropy in a thermally isolated Hamiltonian system apply, i.e., $S\left(\rho_{T}\right)=S\left(\rho_{0}\right)$ and $\Delta F(\alpha) \equiv F_{T}(\alpha)-F_{0}(\alpha)$ is the change in the Helmholtz free energy of the system. Work inequality can be achieved as: $W \geq \Delta F(\alpha)-\alpha^{-1} D_{K L}\left[\rho_{0} \| \rho_{c a n, 0}(\alpha)\right] \equiv W_{L B}(\alpha)$ , where the lower bound for the work denoted as $W_{L B}(\alpha)$. Thus, there exists a best value for $\alpha$; namely, the value for which $W_{L B}(\alpha)$ is a maximum. 